\title{
On Sistotremastrum and similar-looking taxa (Trechisporales, Basidiomycota)
}

\author{
Viacheslav Spirin ${ }^{1}$ (1) $\cdot$ Sergey Volobuev ${ }^{2} \cdot$ Ilya Viner $^{1} \cdot$ Otto Miettinen $^{1} \cdot$ Josef Vlasák $^{3} \cdot$ Nathan Schoutteten $^{4}$. \\ Viviana Motato-Vásquez ${ }^{5} \cdot$ Heikki Kotiranta $^{6} \cdot$ Hernawati $^{7,8} \cdot$ Karl-Henrik Larsson $^{9,10}$
}

Received: 20 October 2020 / Revised: 28 December 2020 / Accepted: 2 February 2021

(C) The Author(s) 2021

\begin{abstract}
The taxonomy of Sistotremastrum (Trechisporales, Basidiomycota) is revised based on morphology and DNA data. The genus is shown to be polyphyletic, and therefore it is split into two units - Sistotremastrum s. str. and Sertulicium, gen. nov. (typified with Corticium niveocremeum). Sistotremastrum s. str. is retained for eleven species of which eight are described as new while Sertulicium encompasses at least six species, including one new to science. Both of these genera are only distantly related to other representatives of the Trechisporales. Additionally, a new poroid neotropical species, Porpomyces abiens (Hydnodontaceae), is described as morphologically similar to some members of Sistotremastrum s. str.
\end{abstract}

Keywords Corticioid fungi $\cdot$ Phylogeny $\cdot$ Taxonomy $\cdot$ New taxa

\section{Introduction}

Sistotremastrum J. Erikss. is a genus of corticioid fungi typified with S. suecicum Litsch. ex J. Erikss. (Eriksson 1958). Initially introduced for two species, it has been gradually expanded to encompass smooth, effused basidiomycetes with clavate basidia producing four to six sterigmata and smooth, thin-walled, inamyloid basidiospores (Boidin and Gilles 1994; Telleria et al. 2013a, 2014). Recently, Gruhn et al. (2018) described two new Sistotremastrum species with a hydnoid hymenophore and constantly four-sterigmatic basidia

Section Editor: Yu-Cheng Dai

Viacheslav Spirin

viacheslav.spirin@helsinki.fi

1 Finnish Museum of Natural History, University of Helsinki, PO Box 7, 00014 University of Helsinki, Helsinki, Finland

2 Komarov Botanical Institute RAS, 2 Prof. Popov str, St. Petersburg 197376, Russia

3 Biology Centre, Academy of Sciences of the Czech Republic, Branišovská 31, 37005 České Budějovice, CZ, Czech Republic

4 Research Group Mycology, Department of Biology, Ghent University, K.L. Ledeganckstraat 35, B-9000 Gent, Belgium

5 Núcleo de Pesquisa em Micologia, Instituto de Botânica, Av. Miguel Estéfano 3687, São Paulo, SP 04301-902, Brazil and thus changed the present concept of the genus. As a consequence, the morphological delimitation of Sistotremastrum from Brevicellicium K.H. Larsson \& Hjortstam and, partly, from Trechispora P. Karst. became obscure. Another yet persisting problem is the separation of Sistotremastrum from Paullicorticium J. Erikss., which has a similar set of microscopic characters (Eriksson et al. 1984). Oberwinkler (1965) moved Sistotremastrum niveocremeum (Höhn. \& Litsch.) J. Erikss., one of the original species, to Paullicorticium, stressing similarities in basidial development. Larsson et al. (2004) included Paullicorticium ansatum Liberta in

6 Finnish Environment Institute, Latokartanonkaari 11, 00790 Helsinki, Finland

7 The Herbarium (ANDA), Universitas Andalas, Padang, Sumatera Barat 25163, Indonesia

8 Fakultas Kehutanan (Faculty of Forestry), Universitas Muhammadiyah Sumatera Barat, Padang, Sumatera Barat 25172, Indonesia

9 Natural History Museum, University of Oslo, PO Box 1172, Blindern, 0318 Oslo, Norway

10 Gothenburg Global Biodiversity Centre, Post Box 461, 40530 Gothenburg, Sweden 
phylogenetic analyses of Agaricomycetes but were unable to place it in one of the clades currently recognized as orders. On the other hand, all Sistotremastrum species, including the generic type, have been found to be members of the Trechisporales (Larsson 2007; Telleria et al. 2013a, 2014; Gruhn et al. 2018). However, most Paullicorticium species have not yet been studied by DNA methods - in particular, $P$. jacksonii Liberta whose connection to Sistotremastrum was discussed in the older literature (Eriksson et al. 1978). In the present paper, we revise the generic limits of Sistotremastrum and similar-looking taxa, and investigate species diversity based on collections from different regions of Eurasia, as well as from North and South America.

\section{Materials and methods}

\section{Morphological study}

Type specimens and collections from herbaria $\mathrm{H}, \mathrm{O}, \mathrm{LE}, \mathrm{S}, \mathrm{GB}$, MA, W, GENT, TRTC, LY, CWU, ANDA, MAN, SP, MG, SING, as well as from the private herbarium of the author JV were studied. Herbarium acronyms are given according to Thiers (2019). Microscopic methods follow Miettinen et al. (2018). All measurements were made from microscopic slides mounted in Cotton Blue (abbreviated as CB in descriptions below), using phase contrast and oil immersion lens (Leitz Diaplan microscope, $\times 1250$ amplification). In total, $20-30$ basidiospores, 20 basidia and subhymenial/subicular hyphae, and at least 10 basidioles and hyphidia were measured for each specimen studied. The following abbreviations are used in morphological descriptions: Lmean basidiospore length, $\mathrm{W}$ - mean basidiospore width, $\mathrm{Q}$ '-length/width ratio, Q - mean length/width ratio, $\mathrm{n}$ - number of measurements per specimens.

\section{DNA extraction, amplification, and sequencing}

DNA was extracted from herbarium specimens using the EZNA Forensic DNA kit (Omega Bio-tek) and AxyPrep Multisource Genomic DNA Miniprep kit (Axygen Biosciences, CA, USA) according to the manufacturer's instructions, except that $50 \mu \mathrm{l}$ of the elution buffer was used in the elution step of the last one. The ribosomal ITS1-5.8SITS2 region was amplified and sequenced with the fungal specific primers ITS1F and ITS4B (Gardes and Bruns 1993). Sequences of nrLSU-rDNA were generated using primers LR0R, CTB6, LR5, and LR7 (Vilgalys and Hester 1990; Haynes et al. 1995). PCR products were visualized using agarose gel electrophoresis and Gel Red staining, and subsequently purified with the Fermentas Genomic DNA Purification Kit (Thermo Fisher Scientific, MA, USA). The resulting products were sequenced with an ABI model 3130 genetic analyzer (Applied Biosystems, CA, USA) and
BigDye v.3.1 and ABI3730XL analyzer (Applied Biosystems) by Macrogen. The raw data were edited and assembled in MEGA 6 (Tamura et al. 2013).

\section{Phylogenetic analyses}

For this study, we generated $60 \mathrm{nrITS}$ and $36 \mathrm{nrLSU}$ sequences. All other sequences used in the analyses were downloaded from GenBank (Benson et al. 2018) or UNITE (Nilsson et al. 2018) (Table 1). All newly generated sequences were deposited in the INSDC.

Extremely high diversity of ITS sequences in the studied taxa precludes attempts to construct a reliable all-encompassing alignment for all Sistotremastrum spp. However, we could distinguish five alignable groups: $S$. niveocremeum complex, S. suecicum and its closest relatives, $S$. aculeocrepitans and its closest relatives, S. fibrillosum complex, and S. rigidum-vigilans clade. We found it reasonable to exclude $S$. fibrillosum, as well as newly described $S$. rigidum and $S$. vigilans from ITS analysis since molecular evidence provided by nrLSU analysis is sufficient for the purpose of our study. Therefore, we generated four alignments for this study: (1) nrLSU-alignment for the Trechisporales, (2) nrITS-alignment for S. niveocremeum, (3) nrITS-alignment limited to S. suecicum and its closest relatives, and (2) nrITSalignment for $S$. aculeocrepitans and its closest relatives. The alignments were calculated through MAFFT 7.429 online server (https://mafft.cbrc.jp/alignment/server/) using the L-INS-I strategy (Katoh et al. 2017) and then manually adjusted. The alignments are deposited in TreeBASE (S25768).

For the genus-level analysis, a nrLSU dataset (1) was assembled with representatives of Trechisporales (65 sequences). The resulting tree was rooted with Onnia leporina and Sphagnomphalia brevibasidiata (Hymenochaetales) and Sphaerobolus stellatus (Geastrales, Phallomycetidae). This choice was guided by the current JGI Basidiomycota tree (https://mycocosm.jgi.doe.gov/mycocosm/species-tree/tree; FJDxL? organism=basidiomycota) where Trechisporales is recovered close to Hymenochaetales and Phallomycetidae. After removing unalignable, ambiguous alignment positions, the alignment length was 742 bp with 184 variable site patterns. The three ITS datasets were assembled similarly: the $S$. niveocremeum species complex alignment (2) contained 60 sequences and had the length of 329 bp with 40 variable site patterns after removing unalignable, ambiguous positions. The $S$. suecicum species complex analysis (3) contained 22 sequences, with alignment length of $508 \mathrm{bp}$ with 13 variable site patterns after removing ambiguous positions. The $S$. aculeocrepitans species complex analysis (4) contained 22 sequences with the alignment length of $470 \mathrm{bp}$ with 37 variable site patterns. All the ITS trees are midpoint-rooted.

We inferred phylogenetic trees with maximum likelihood (ML), maximum parsimony (MP), and Bayesian inference (BI) but show here only the latter since all trees show 
Table 1 Sequences generated for the present study

\begin{tabular}{|c|c|c|c|c|c|}
\hline \multirow[t]{2}{*}{ Species } & \multirow[t]{2}{*}{ Specimen/herbarium } & \multirow[t]{2}{*}{ Geographic origin (ISO code) } & \multirow[t]{2}{*}{ Host } & \multicolumn{2}{|c|}{ GenBank number } \\
\hline & & & & nrITS & nrLSU \\
\hline Brevicellicium exile & Spirin $8370(\mathrm{H})$ & US-WA & Tsuga heterophylla & MT002322 & MT002338 \\
\hline B. olivascens & Spirin $4446(\mathrm{H})$ & RU-NIZ & Quercus robur & MT002327 & - \\
\hline B. viridulum & Kotiranta $29271(\mathrm{H})$ & RU-SAK & Alnus hirsuta & MN983275 & - \\
\hline Luellia cystidiata & Læssøe 13875 (GB) & DK & Picea abies & MW371211 & MW371211 \\
\hline Porpomyces abiens & Vlasák 1808/16 (H) & GF & Hardwood & MN987945 & MN987945 \\
\hline P. abiens & Vlasák 1808/39 (H) & GF & Hardwood & MN987942 & MN987942 \\
\hline Pteridomyces galzinii & Bernicchia $8122(\mathrm{~GB})$ & IT & Polystichum aculeatum & MN937559 & MN937559 \\
\hline Sertulicium granuliferum & Spirin $9296(\mathrm{H})$ & RU-NIZ & Populus tremula & MT002321 & - \\
\hline S. granuliferum & LE 292194 & RU-ORL & Betula pendula & MT002325 & MT002333 \\
\hline S. granuliferum & LE 314053 & RU-ORL & Q. robur & MT002313 & MT002340 \\
\hline S. granuliferum & LE 314054 & RU-ORL & Q. robur & MT002315 & MT002342 \\
\hline S. granuliferum & LE 314055 & RU-ORL & B. pendula & MT002314 & MT002341 \\
\hline S. granuliferum & LE 314056 & RU-ORL & Q. robur & MT002316 & MT002343 \\
\hline S. granuliferum & LE 314057 & RU-ORL & Q. robur & MT002312 & MT002339 \\
\hline S. granuliferum & Kotiranta $26776(\mathrm{H})$ & RU-TY & Larix sibirica & MT002323 & - \\
\hline S. granuliferum & CWU 4704 & UA & Salix alba & MT002326 & - \\
\hline S. granuliferum & Miettinen $14813.2(\mathrm{H})$ & US-MA & Cut bole & MT075854 & - \\
\hline S. granuliferum & Miettinen $16083(\mathrm{H})$ & US-MA & Hardwood & MT002319 & - \\
\hline S. granuliferum & Miettinen $17272(\mathrm{H})$ & US-MA & Quercus sp. & MT002330 & - \\
\hline S. granuliferum & Larsson 12282 (GB) & US-TN & Decayed wood & MN937560 & MN937560 \\
\hline S. granuliferum & Larsson 12297 (GB) & US-TN & Decayed wood & MN937561 & MN937561 \\
\hline S. jacksonii & Svantesson $699(\mathrm{O})$ & NO & Picea abies & MN937562 & MN937562 \\
\hline S. jacksonii & Spirin $10425(\mathrm{H})$ & RU-LEN & P. abies & MN987943 & MN987943 \\
\hline S. jacksonii & Miettinen $17141(\mathrm{H})$ & US-NY & Abies sp. & MN987944 & - \\
\hline S. lateclavigerum & Spirin 13457 & SI & P. abies & MW049161 & - \\
\hline S. niveocremeum & Van Autgaerden S-20 (GENT) & $\mathrm{BE}$ & Hardwood & MN947227 & MN930920 \\
\hline S. niveocremeum & Miettinen $14925.3(\mathrm{H})$ & FI & Sorbus aucuparia (?) & MT075855 & - \\
\hline S. niveocremeum & Söderholm 4050 (H) & FI & Salix sp. & MT075856 & MT002334 \\
\hline S. niveocremeum & Larsson 13727 (GB) & FR & Hardwood & MN937563 & MN937563 \\
\hline S. niveocremeum & Kotiranta $26267(\mathrm{H})$ & RU-KRA & Alnus sibirica & MT075858 & MT002337 \\
\hline S. vernale & Söderholm $3886(\mathrm{H})$ & FI & P. abies & MT002311 & MT664174 \\
\hline Sertulicium sp. 1 & Spirin $5158(\mathrm{H})$ & RU-KHA & Corylus mandshurica & MT075857 & MT002344 \\
\hline Sistotremastrum aculeatum & Miettinen $10380.1(\mathrm{H})$ & $\mathrm{CN}-\mathrm{YN}$ & Hardwood (?) & MN991176 & MW045423 \\
\hline S. aculeatum & Miettinen 13799.1 (ANDA) & ID-SB & Hardwood & MN988623 & - \\
\hline S. aculeatum & Dunaev KUN 1105 (H) & TH & Hardwood & MN991181 & - \\
\hline S. aculeicrepitans & Larsson 16097 (URM) & BR-PB & Hardwood & MN937564 & MN937564 \\
\hline S. aculeicrepitans & Larsson 16478 (MG) & BR-PA & Hardwood & MN937565 & - \\
\hline S. confusum & Larsson 16004 (URM) & BR-PE & Hardwood & MN937567 & MN937567 \\
\hline S. confusum & Larsson 16023 (URM) & BR-PE & Hardwood & MN937566 & - \\
\hline S. denticulatum & Motato-Vásquez 894 (SP) & BR-SP & Fallen branch & MN954694 & MW045424 \\
\hline S. fibrillosum & Larsson 16988 (MG) & BR-PA & Hardwood & MN937568 & MN937568 \\
\hline S. geтіпит & Miettinen 14333 (MAN) & ID-PB & Intsia bijuga & MN991177 & MN991177 \\
\hline S. induratum & Spirin $8598(\mathrm{H})$ & US-WA & Abies grandis & MT002324 & MT664173 \\
\hline S. induratum & Spirin $8804(\mathrm{H})$ & US-WA & Picea sitchensis & MT002318 & - \\
\hline S. mendax & J. Nordén 9579 (O) & $\mathrm{NO}$ & P. abies & MN937569 & - \\
\hline S. mendax & Larsson $12022(\mathrm{O})$ & NO & P. abies & MN937570 & MN937570 \\
\hline S. mendax & Miettinen $20946(\mathrm{H})$ & RU-LEN & P. abies & MN991179 & - \\
\hline
\end{tabular}


Table 1 (continued)

\begin{tabular}{|c|c|c|c|c|c|}
\hline \multirow[t]{2}{*}{ Species } & \multirow[t]{2}{*}{ Specimen/herbarium } & \multirow[t]{2}{*}{ Geographic origin (ISO code) } & \multirow[t]{2}{*}{ Host } & \multicolumn{2}{|c|}{ GenBank number } \\
\hline & & & & nrITS & nrLSU \\
\hline S. rigidum & Motato-Vásquez 833 (SP) & BR-SP & Fallen log & MN954693 & MW045435 \\
\hline S. suecicum & Spirin $8932(\mathrm{H})$ & CA-BC & Pinus contorta & MT002317 & - \\
\hline S. suecicum & Kunttu $5959(\mathrm{H})$ & FI & Pinus sylvestris & MT075859 & MT002335 \\
\hline S. suecicum & LE 295792 & RU-ARK & P. sylvestris & MT002332 & - \\
\hline S. suecicum & Miettinen $14550.1(\mathrm{H})$ & SE & P. sylvestris & MT075860 & MT002336 \\
\hline S. suecicum & Larsson 11849 (GB) & SE & P. abies (?) & MN937571 & MN937571 \\
\hline S. suecicum & Miettinen $14829(\mathrm{H})$ & US-MA & Fallen branch & MT075861 & - \\
\hline S. suecicum & Miettinen $16061(\mathrm{H})$ & US-MA & Tsuga canadensis & МT002328 & - \\
\hline S. suecicum & Miettinen $16618(\mathrm{H})$ & US-MA & Tsuga sp./Pinus sp. & MT002331 & - \\
\hline S. vigilans & Fonneland 2011-78 (O) & NO & P. abies & MN937572 & MN937572 \\
\hline S. vigilans & Spirin $10097(\mathrm{H})$ & RU-LEN & P. abies & MN991178 & - \\
\hline S. vigilans & Spirin $8778(H)$ & US-WA & T. heterophylla & MN991182 & MN991182 \\
\hline Suillosporium cystidiatum & Spirin $3830(\mathrm{H})$ & RU-KHA & Picea ajanensis & MN937573 & MN937573 \\
\hline
\end{tabular}

congruity of the phylogenetic signal. The best nucleotide substitution model was chosen with TOPALi 2.5 (Milne et al. 2008) based on the Akaike information criterion (AIC), which were $\mathrm{GTR}+\mathrm{G}+\mathrm{I}$ (nst $=6$, rates = invgamma) for the nrLSUdataset, GTR $+\mathrm{G}$ (nst $=6$, rates $=$ gamma) for the $S$. niveocremeum dataset, and F81 (nst = 1) for the S. suecicum dataset. The suggested models were implemented in the Bayesian phylogenetic analyses. We performed Bayesian inference with MrBayes 3.2 (Ronquist et al. 2012). In the analyses, three parallel runs with four chains each, temp $=0.2$, were run for 3 million generations. All chains converged to $<0.01$ average standard deviation of split frequencies. A burn-in of $25 \%$ was used in the final analyses. Maximum likelihood (ML) analysis was performed in RAxML 7.2.8 (Stamatakis 2006) implemented in Geneious version 9.1.8 (http://www.geneious.com) (Kearse et al. 2012). Following models suggested by TOPALi 2.5, we preferred to use the GTR model with gamma correction (GTR GAMMA) in ML analysis. Bootstrapping was performed using the "Rapid bootstrapping" algorithm with the number of bootstrap replicates set as 1000. Maximum parsimony (MP) analysis was performed using SeaView version 4 (Gouy et al. 2010) with the ignoring all gap sites option. The number of bootstrap replicates was set as 1000 .

\section{Results}

The nrLSU dataset encompasses all known genera of the Trechisporales as defined by Larsson et al. (2004) and Larsson (2007), with addition of Pteridomyces and Suillosporium. The overall topologies of the Trechisporales were highly similar (Fig. 1). They split the order into three strongly supported clades:

(A) the Sistotremastrum s. str. subclade ( $\mathrm{pp}=1$, bs $=99 \%)$ includes the generic type, $S$. suecicum, and two unnamed closely related species (introduced below as $S$. induratum and $S$. mendax), all with six-sterigmatic basidia, plus eight more distantly related species with four-sterigmatic basidia ( $S$. aculeocrepitans, S. fibrillosum and six new species);

(B) the $S$. niveocremeum subclade ( $\mathrm{pp}=1$, bs $=100 \%$ ) covers five species formerly considered as members of Sistotremastrum (except $P$. jacksonii and one unnamed taxon) but separated below into a new genus, Sertulicium. Morphological arguments for this solution are given in the taxonomic part of the present paper. Sistotremastrum guttuliferum is a younger synonym of Trechispora granulifera, and therefore designated as Sertulicium granuliferum in the phylogenetic trees; and

(C) the Hydnodontaceae clade ( $\mathrm{pp}=1$, bs $=99 \%$ ) covers ten genera currently recognized in the family (Larsson 2007; Liu et al. 2019). Among them, two sequences belong to an unnamed poroid fungus from French Guiana introduced below as Porpomyces abiens. Anatomically, its basidiocarps are confusingly similar to the four-sterigmatic Sistotremastrum species (i.e., S. aculeocrepitans, $S$. fibrillosum, and a number of new species described in this study) and differ from them mainly by a truly poroid hymenophore and smaller basidiospores. Sequences of three Brevicellicium species (including the generic type, B. exile) showing considerable morphological similarity to the four-sterigmatic Sistotremastrum species are also resolved in the Hydnodontaceae, in accordance to earlier 


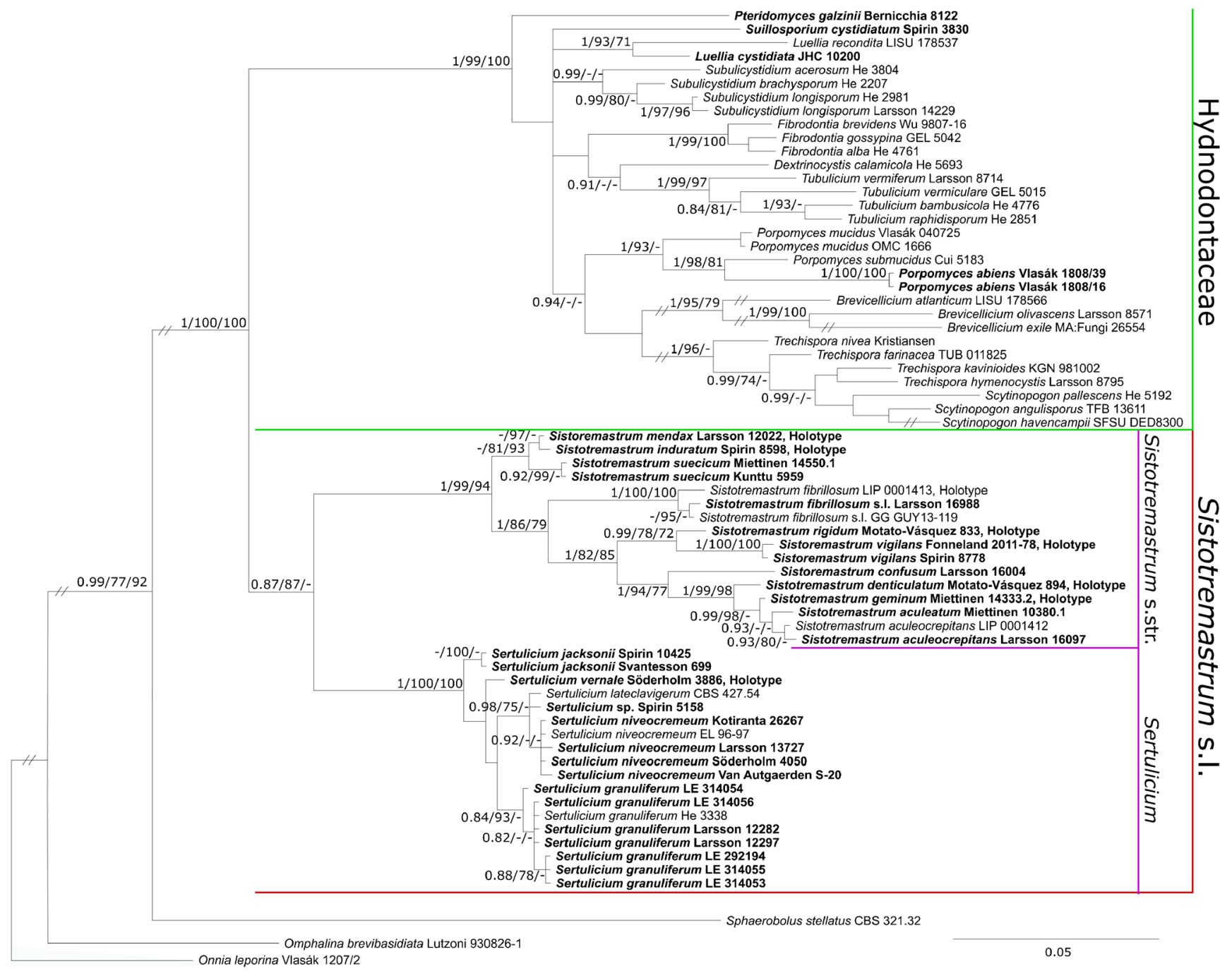

Fig. 1 Phylogenetic relationships of the Trechisporales inferred from nrLSU sequences using Bayesian analysis. A 50\% majority rule consensus phylogram. Bayesian posterior probabilities, ML bootstrap,

studies (Larsson 2007; Telleria et al. 2013b). Morphological traits differentiating Sistotremastrum s. str. from Brevicellicium and Trechispora are discussed in the taxonomic part of this paper. As for the family-level rearrangement of Sistotremastrum and Sertulicium, we address this question to further studies with the use of additional genetic markers.

Additionally, three ITS datasets were constructed for the new genus Sertulicium and the Sistotremastrum suecicum and S. aculeocrepitans complexes to clarify species limits with the use of larger amount of ITS sequences. The ITS-based topology of Sertulicium (Fig. 2) indicates a presence of up to ten species in the genus of which we have named six. Furthermore, ITS sequences of $S$. jacksonii and $S$. vernale reveal some differences (up to 1.4\%) between European and and MP bootstrap values are shown on nodes; branch lengths reflect estimated number of changes per site

extra-European material. This may indicate that both species, as they are here delimited, actually contain more than one taxon. However, more samples and genetic markers are needed to solve this problem. The second ITS-based phylogeny is restricted to the S. suecicum complex (Fig. 3). It shows the presence of at least two more species (described below as $S$. induratum and $S$. mendax) morphologically similar and phylogenetically close to the generic type of Sistotemastrum. The third ITS-based phylogram shows phylogenetic relationships of S. aculeocrepitans and up to five closely related species. Of them, three are described as new to science, all having four-sterigmatic basidia (S. aculeatum, S. denticulatum, and S. geminum) (Fig. 4). Four remaining Sistotremastrum species (S. confusum, S. fibrillosum, S. rigidum, and S. vigilans) dealt with in the present paper are represented in the nrLSU phylogeny (Fig. 1). Their ITS sequences are available in GenBank (Table 1). Sequenced specimens are marked by asterisk. 


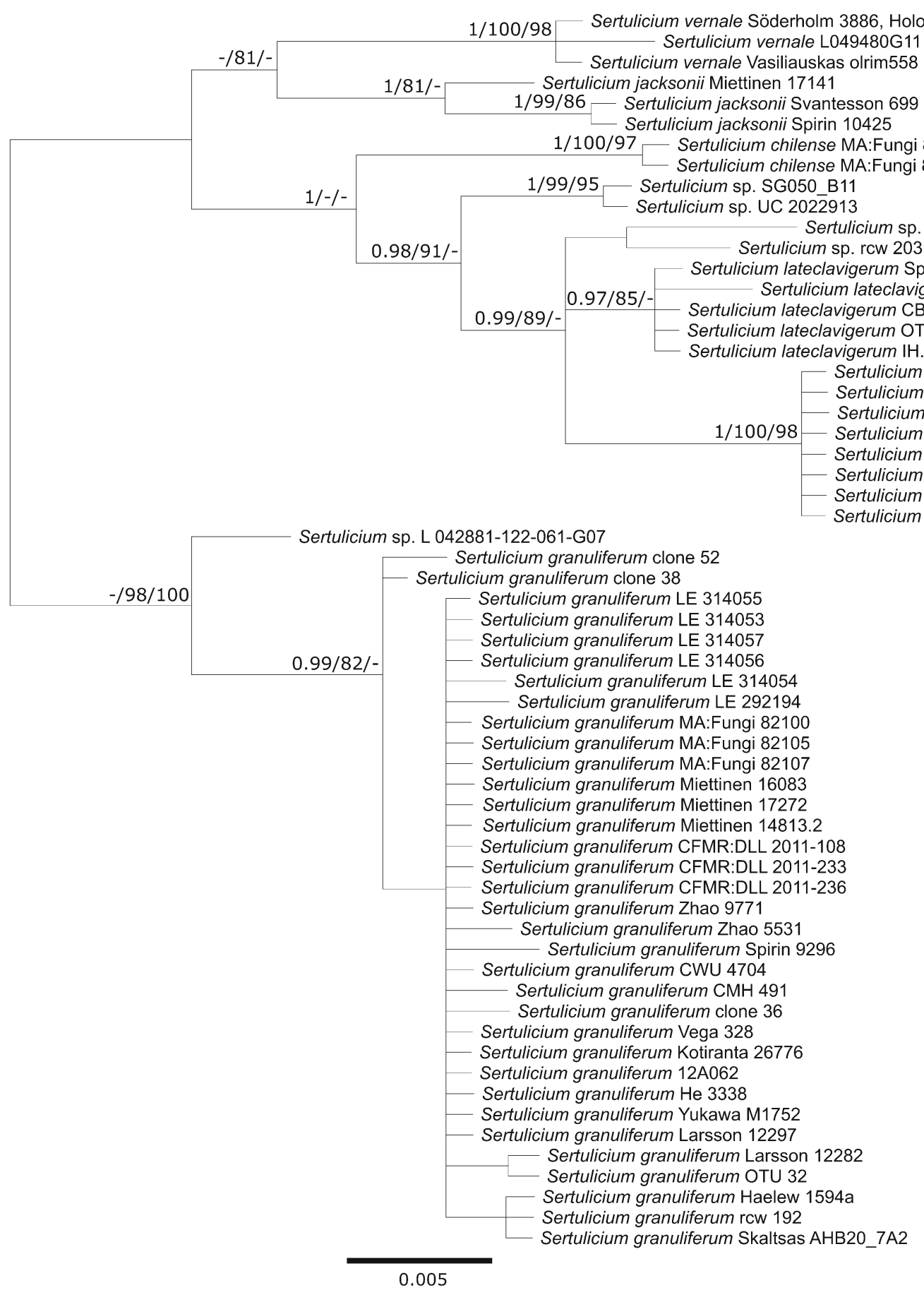

Fig. 2 Phylogenetic relationships of Sertulicium inferred from ITS sequences using Bayesian analysis. A 50\% majority rule consensus phylogram. Bayesian posterior probabilities, ML bootstrap, and MP bootstrap values are shown on nodes; branch lengths reflect estimated number of changes per site

Zhao, was recently described from China (Wu et al. 2015). Here, we introduce the third representative of the genus, $P$. abiens from South America. From P. mucidus and P. submucidus, $P$. abiens differs by distinctly thick-walled and tightly arranged hyphae in subiculum and trama. This hyphal structure is clearly different from the loosely arranged, rather delicate, thin- or only slightly thick-walled hyphae of 
Fig. 3 Phylogenetic relationships of Sistotremastrum suecium and closely related taxa inferred from ITS sequences using Bayesian analysis. A 50\% majority rule consensus phylogram. Bayesian posterior probabilities, ML bootstrap, and MP bootstrap values are shown on nodes; branch lengths reflect estimated number of changes per site
Fig. 4 Phylogenetic relationships of Sistotremastrum aculeocrepitans and closely related taxa inferred from ITS sequences using Bayesian analysis. A 50\% majority rule consensus phylogram. Bayesian posterior probabilities, ML bootstrap, and MP bootstrap values are shown on nodes; branch lengths reflect estimated number of changes per site
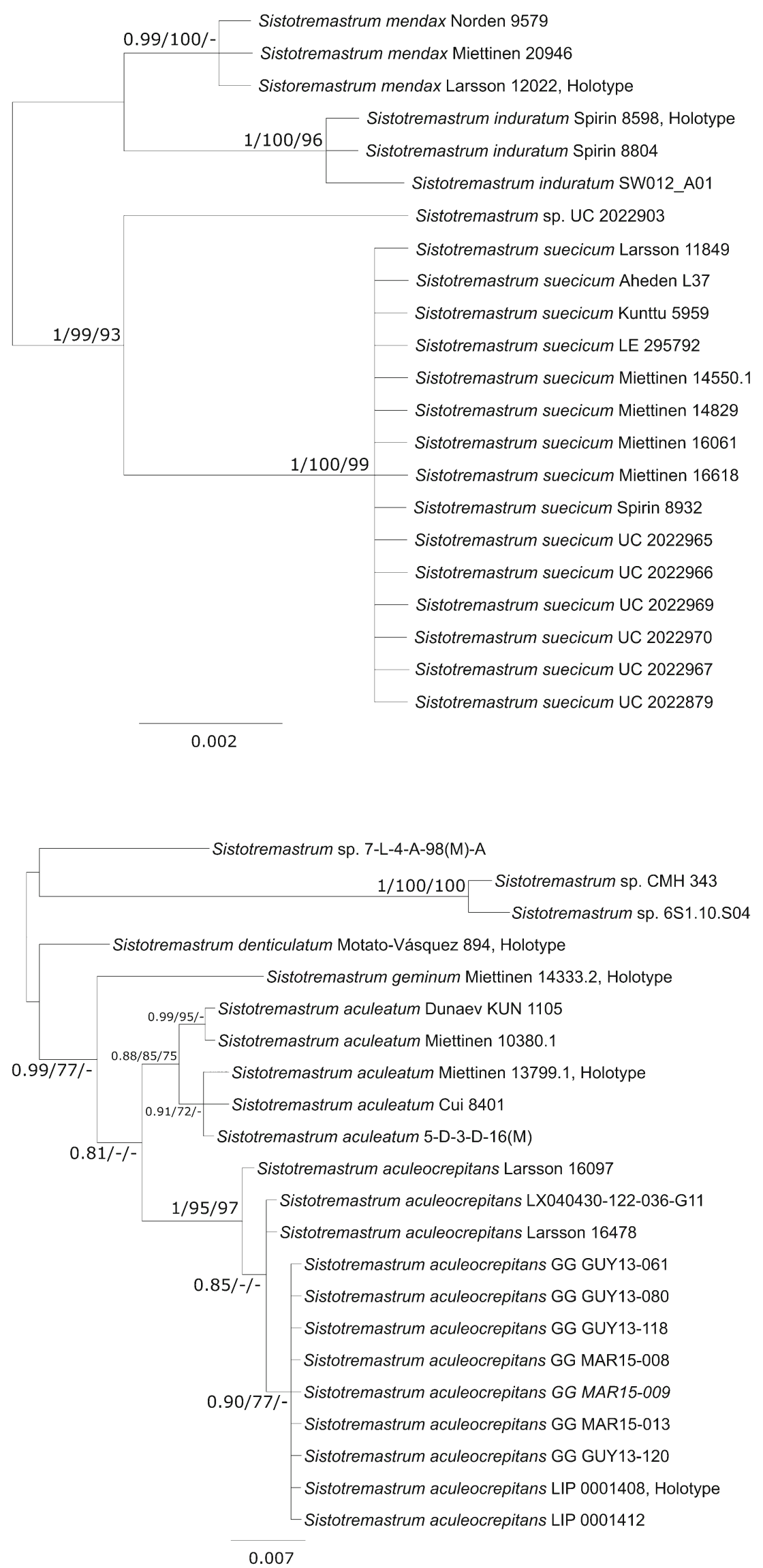
P. mucidus and P. submucidus. The two latter species have thin-walled basidia and no sterile hymenial elements while basidia are slightly thick-walled in a senescent hymenium of $P$. abiens and hyphidia are present. Rather tough basidiocarps consisting of predominantly thick-walled hyphae and short, tardily thick-walled basidia of $P$. abiens are reminiscent of hydnoid-semiporoid Sistotremastrum species, i.e., S. aculeatum, S. aculeocrepitans, and S. denticulatum. The only reliable anatomical traits differentiating them from $P$. abiens are their suburniform basidia and somewhat larger basidiospores. Moreover, no truly poroid species are so far known in Sistotremastrum s. str.

Porpomyces abiens Vlasák \& Spirin, sp. nov.-Figs. 5 and 6

MB 833939

Holotype. French Guiana. Remire-Montjoly: Lac du Rorota, rotten angiosperm wood, 23.VIII.2018 Vlasák 1808/16* (H 7009714).

Etymology. Abiens (Lat., part. from abeo)—departing, deviating.

Basidiocarps effused, first soft-floccose, then rather tough, covering several $\mathrm{cm}, 0.2-1 \mathrm{~mm}$ thick. Hymenial surface pale cream-colored to yellowish or pale ochraceous, poroid, pores angular to sinuous, partly fusing together, strongly elongated on sloping substrate, 6-9 per $\mathrm{mm}$, with thin, entire dissepiments. Margin white to pale cream-colored, fibrillose or compact, in some portions with thin, white, pronounced hyphal strands.

Hyphal structure monomitic; hyphae clamped. Subicular hyphae slightly to distinctly thick-walled, (2.2-) 2.7-7.3 (-7.8) $\mu \mathrm{m}$ in diam. $(\mathrm{n}=20 / 1)$, interwoven or in subparallel bundles, often distinctly inflated (up to $10 \mu \mathrm{m})$; some hyphae encrusted by densely distributed thorn-like crystals or subglobose resinous globules. Tramal hyphae distinctly thick-walled, rather tightly arranged, interwoven to subparallel, occasionally anastomosing, partly glued together, (2.2-) 2.8-5.8 (-6.4) $\mu \mathrm{m}$ in diam. $(n=40 / 2)$, sometimes inflated near septa; some hyphae encrusted by densely distributed subglobose resinous globules. Subhymenial hyphae thin- to slightly thick-walled, mostly short-celled and distinctly inflated, (2.0-) 2.2-5.2 (-5.4) $\mu \mathrm{m}$ in diam. $(\mathrm{n}=40 / 2)$. Rhomboid or prismatic crystals occasionally present among subicular or tramal hyphae, up to $15 \mu \mathrm{m}$ in widest dimension, solitary or in large groups. Hyphidia rarely present, 10-12 $\times$ 3.5-5 $\mu \mathrm{m}$. Basidia subglobose to barrel-shaped or shortly clavate, 2-4-spored, (4.8-) 4.9-7.9 (-8.0) × (3.4-) 3.7$4.8(-5.0) \mu \mathrm{m}(\mathrm{n}=30 / 2)$, senescent basidia slightly thickwalled. Basidioles subglobose to globose, 4-5 × 4-4.5 $\mu \mathrm{m}$. Basidiospores broadly cylindrical to ellipsoid, more rarely somewhat tapering to the apex (lacrymoid), (2.3-) $2.5-3.2(-3.3) \times(1.6-) 1.7-2.2(-2.3) \mu \mathrm{m}(\mathrm{n}=60 / 2), \mathrm{L}=$
2.87-2.94, $\mathrm{W}=1.98-2.00, \mathrm{Q}=1.46-1.47$, contents homogeneous and $\mathrm{CB}(+)$.

Remarks. Porpomyces abiens has been detected in three localities in French Guiana. It seems to be a saprotrophic species occurring on wood remnants, various debris and soil.

Specimens examined. French Guiana. Remire-Montjoly: Lac du Rorota, hardwood, 23.VIII.2018 Vlasák 1808/39* (H, JV). Roura: Natural Reserve of Kaw, Patawa Lodge, on soil between the roots of uprooted tree, 31.VIII.2018 Vlasak 1808/146 (JV), Amazon Lodge, rotten hardwood log, 4.IX.2019 Vlasák 1909/29*(JV).

Sertulicium Spirin, Volobuev \& K.H. Larss., gen. nov. MB 833941

Etymology. Sertulum (Lat., noun) - a small chaplet.

Basidiocarps effused, very thin to rather thin (usually up to $0.1 \mathrm{~mm}$ thick), pruinose or waxy. Hymenophore smooth. Hyphal structure monomitic; all hyphae clamped, CB (+). Cystidia mostly absent, hyphidia rarely present, simple. Basidia clavate, with 4-6 sterigmata, normally not collapsing at the apex. Basidiospores thin-walled (but the wall distinct), narrowly ellipsoid to cylindrical, inamyloid, acyanophilous, contents homogeneous and CB (+). On rotten wood of deciduous trees and conifers.

Generic type. Corticium niveocremeum Höhn. \& Litsch.

Morphological differences between Sertulicium and Sistotremastrum s. str. cannot be easily grasped due to the extreme anatomical simplicity of their representatives. Nevertheless, some distinguishing characters are indicated here awaiting more in-depth analysis in the future. First, all but one Sertulicium spp. are extremely thin fungi consisting of a few subicular hyphae and the overlying subhymenium. The subicular hyphae are scattered; i.e., they do not produce hyphal strands so characteristic for most Sistotremastrum s. str. species studied by us. The only exception is Sertulicium granuliferum, having the most elaborate fructifications in the genus. Its basidiocarps start their development as radially arranged hyphal bundles becoming quickly covered by randomly arranged hyphae and finally indiscernible. In contrast, in Sistotremastrum s. str. subicular hyphal strands can be detected even in senescent basidiocarps if hyphae are not totally collapsed. Second, basidia in all Sertulicium spp. bear up to six sterigmata while at least some Sistotremastrum s. str. species are strictly four-sterigmatic. Moreover, apical parts of basidia in the latter genus often collapse, and hymenial cells of this kind dominate in well-developed and in senescent basidiocarps. Such apically collapsing basidia may occur in Sertulicium spp., too, but they are as a rule rare. Finally, all Sertulicium spp. have a smooth hymenial surface while about a half of the currently 
Fig. 5 Basidiocarps of a Porpomyces abiens (holotype), b Sertulicium granuliferum (Miettinen 23459), c Sistotremastrum aculeatum (holotype), d S. mendax (Spirin 11208), e S. suecicum (Miettinen 13310), and $\mathbf{f} S$. suecicum (senescent basidiocarps) (Miettinen 14548)
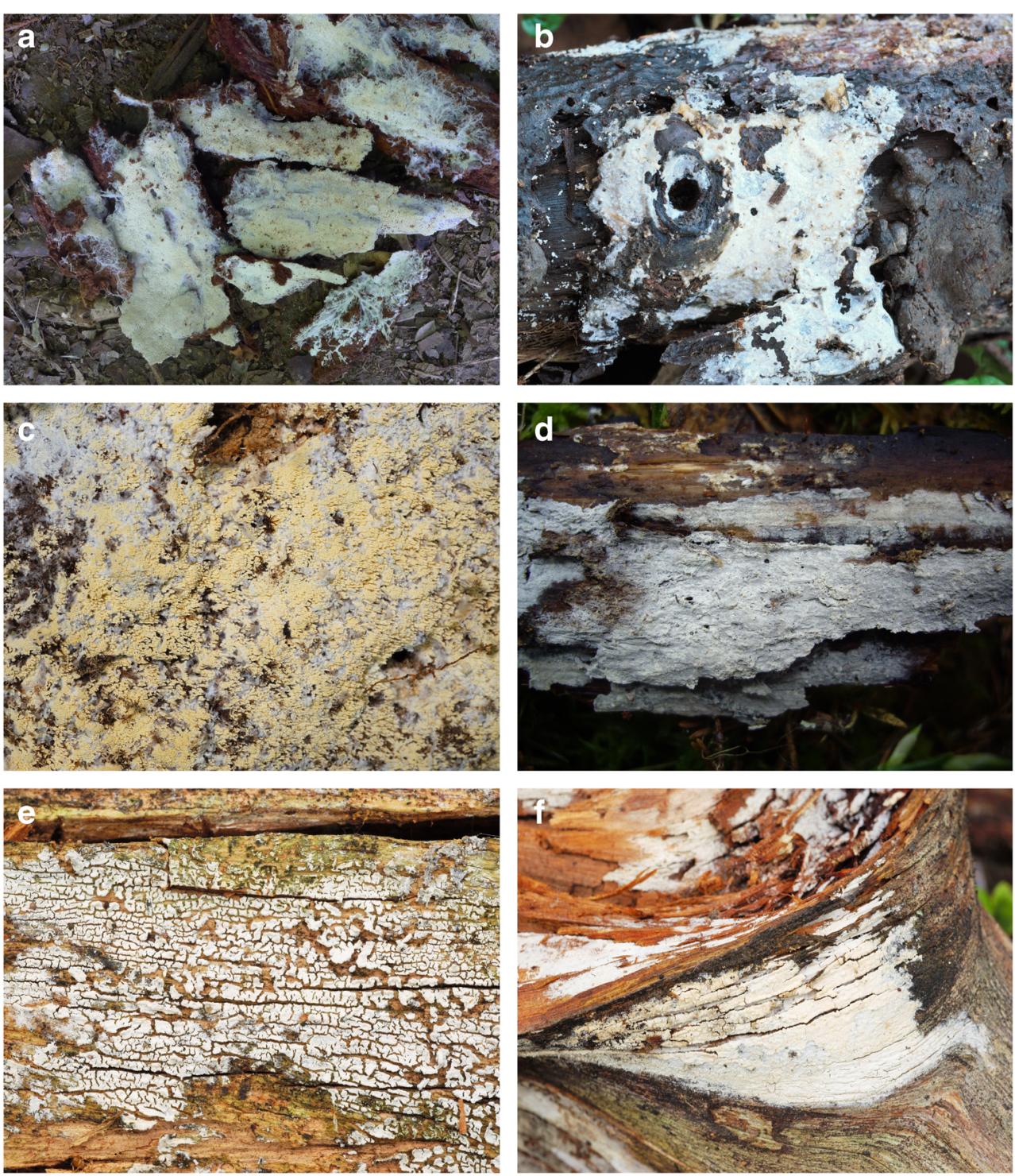

known Sistotremastrum spp. have a hydnoid hymenophore.

Sertulicium chilense (Telleria, M. Dueñas \& M.P. Martín) Spirin \& Volobuev, comb. nov.

$\equiv$ Sistotremastrum chilense Telleria, M. Dueñas \& M.P. Martín, Phytotaxa 158: 94, 2014 (as 'chilensis').

MB 833942

Sistotremastrum chilense was described from Patagonia (Telleria et al. 2014) as a relative of S. guttuliferum (see under Sertulicium granuliferum below). From the latter species, it differs primarily in having long-celled subhymenial hyphae (vs. short-celled and somewhat inflated in S. granuliferum). DNA data confirm its placement in Sertulicium.
Sertulicium granuliferum (Hallenb.) Spirin \& Volobuev, comb. nov.-Figs. 5 and 10

MB 833943

$\equiv$ Trechispora granulifera Hallenb., Iranian J. Plant Path. 14: 77, 1978. Holotype. Iran. Gilan: Asalem, Parrotia persica, 16.VII.1976 Hallenberg 1885 (GB 23442, studied).

= Sistotremastrum guttuliferum Melo, M. Dueñas, Telleria \& M.P. Martín, Mycological Progress 12: 688, 2013. Holotype. Portugal. Madeira: Santana, decayed wood, 19.X.2006 Telleria 16566* (MA F82105, studied).

Basidiocarps effused, initially reticulate, soft-pellicular, then continuous, waxy, covering several $\mathrm{cm}, 0.05-0.1 \mathrm{~mm}$ thick. Hymenial surface first cream-colored, later yellowish to pale ochraceous, smooth or indistinctly tuberculate, 


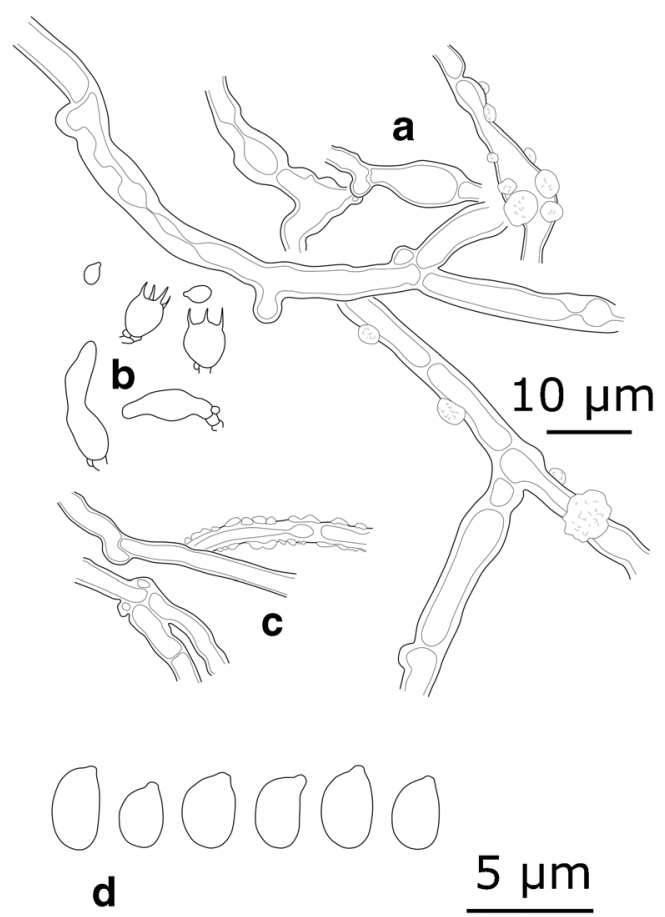

Fig. 6 Microscopic structures of P. abiens (holotype). a Subicular hyphae. b Hymenial cells. c Tramal hyphae. d Basidiospores

occasionally cracking in senescent basidiocarps. Margin first pruinose, later compact and rather sharply delimited. Small white patches spread at the marginal areas of young specimens, consisting of crystal agglomerations.

Hyphal structure monomitic; hyphae clamped, quickly collapsing. Subicular hyphae subparallel (juvenile specimens) to interwoven, thin- or slightly thick-walled, branched mostly at right angles, (3.0-) 3.1-6.5 (-6.9) $\mu \mathrm{m}$ in diam. $(\mathrm{n}=100 / 5)$. Subhymenial hyphae thin-walled, densely arranged, some short-celled and slightly inflated, (2.2-) 2.3-4.2 (-4.6) $\mu \mathrm{m}$ in diam. $(n=100 / 5)$. Hyphidia occasionally present, embedded, simple, 3-4 $\mu \mathrm{m}$ in diam. Basidia clavate, 4-6-spored, gradually tapering to the basal part, (11.3-) 13.6-30.2 (-32.7) $\times(4.3-) 4.4$ $6.6(-6.7) \mu \mathrm{m}(\mathrm{n}=100 / 5)$. Basidioles clavate to bladder-shaped, (7.3-) 9.1-13.8 (-14.6) × (4.7-) 4.8-7.2 (-7.3) $\mu \mathrm{m}(\mathrm{n}=30 / 2)$. Basidiospores cylindrical, straight, rarely slightly curved, (4.8-) 4.9-7.3 (-8.0) × (2.3-) 2.5-3.3 (-3.5) $\mu \mathrm{m}(\mathrm{n}=270 / 9), \mathrm{L}=5.40$ $6.76, \mathrm{~W}=2.78-3.07, \mathrm{Q}=1.81-2.41$.

Remarks. This species was introduced as a member of Trechispora due to some morphological similarity to the smooth-spored members of that genus (i.e., Trechispora cohaerens group) (Hallenberg 1978). Larsson (1992) found clavate basidia with 4-5 sterigmata in the type collection and thus pointed towards possible affinities with Sistotremastrum. After rechecking the type, as well as morphological and DNA studies of newly collected material, we conclude that $T$. granulifera is an older name for the species recently introduced as Sistotremastrum guttuliferum (Telleria et al. 2013a). Consequently, a new combination is proposed.
Sertulicium granuliferum differs from the similar-looking $S$. niveocremeum in having thicker and softer basidiocarps with a well-developed subicular layer and smaller basidiospores (Table 2).

Sertulicium granuliferum is widely distributed in temperate Eurasia. It seems to be not rare along the eastern coast of Canada and the USA, and its distribution in North America stretches to the central part of Mexico. All but one specimen came from wood remnants of deciduous trees, mostly from decayed wood but a few from still corticated, rather tough branches or logs. One specimen from Ethiopia is morphologically indistinguishable from other collections studied by us and therefore accepted here as S. granuliferum. Three other collections from Africa (Kenya and Tanzania) are microscopically identical to $S$. granuliferum but strikingly different macroscopically due to a well-developed, distinctly fibrillose margin. They may represent yet another species in this genus and thus treated as Sertulicium aff. granuliferum under Specimens examined. ITS regions of two published Sistotremastrum genomes (Nagy et al. 2016; Sistotremastrum niveocremeum LOAV00000000.1 and Sistotremastrum suecicum SRA:SRR1800527.68548550.2) appear to be conspecific and belong to Sertulicium granuliferum.

Specimens examined. Belgium. Oost-Vlaanderen: Kluisbergen, Kluisbos, deciduous wood, 20.II.2017 Schoutteten 17-021, 17-022 (GENT), 7.III.2017 Schoutteten 17-108 (GENT). Canada. Québec: Outaouais, Gatineau Nat. Park, angiosperm, 17.X.1967 Eriksson 9155 (GB), Acer sp., 17.X.1967 Eriksson 9177 (GB). Ethiopia. Shoa Prov.: Shashemene, dead hardwood twig, 18.VII.1990 Ryvarden 28557 (O). Germany. Nordrhein-Westphalen: Essen, Werden, deciduous wood, 6.I.2020 Miettinen $23459(\mathrm{H})$. Italy. Lombardy: Varese, Valganna, San Gemolo, Fraxinus excelsior, 14.X.2019 Spirin 13657 (H). Mexico. Vera Cruz: Barranca de Pescado, angiosperm, 26.IX.1985 Ryvarden 23449 (O). Russia. Kemerovo Reg.: Novokuznetsk Dist., Kuzedeevo, Betula sp., 3.IX.2011 Kotiranta 28540 (H), Salix caprea, 4.IX.2011 Kotiranta 28597 (H). Leningrad Reg.: Boksitogorsk Dist., Chagoda, Alnus incana, 9.V.2018 Spirin 11877 (H). Nizhny Novgorod Reg.: Bogorodsk Dist., Krastelikha, Tilia cordata, 14.IX.2010 Spirin 3503 (H), Q. robur, 2.X.2015 Spirin 9898, 9899, 9910 (H), 17.VII.2016 Spirin 10310, 10313, 10315 (H), 5.V.2018 Spirin 11862 (H); Lukoyanov Dist., Panzelka, Padus avium, 17.VIII.2015 Spirin 9479 (H), 9.VIII.2016 Spirin 10577 (H), Razino, Populus tremula, 15.VIII.2015 Spirin 9296* (H), Q. robur, 10.VIII.2016 Spirin $10605(\mathrm{H})$, T. cordata, 23.VII.2018 Spirin 12006 (H), Sanki, Acer platanoides, 20.VIII.2015 Spirin 9658 (H); Pavlovo Dist., Chudinovo, P. tremula, 3.X.2015 Spirin 9977 (H), 15.VII.2016 Spirin 10281, 10284 (H), T. cordata, 15.VII.2016 Spirin 10263 (H). Oryol Reg.: Krasnozorensky Dist., Malinovo, Q. robur, 7.X.2012 Volobuev (LE 314056*); Novoderevenkovsky 
Table 2 Basidiospore dimensions of Porpomyces and Sistotremastrum $\mathrm{s}$. lato species. Species name and basidiospore dimensions for a given taxon are presented in bold

\begin{tabular}{|c|c|c|c|c|c|c|c|}
\hline Species/specimen & $\mathrm{L}^{\prime}$ & $\mathrm{L}$ & $\mathrm{W}^{\prime}$ & W & $\mathrm{Q}^{\prime}$ & Q & $\mathrm{n}$ \\
\hline Porpomyces abiens & (2.3) $2.5-3.2(3.3)$ & 2.91 & (1.6) $1.7-2.2(2.3)$ & 1.99 & (1.2) 1.3-1.7 (1.8) & 1.47 & 60 \\
\hline holotype & (2.4) 2.5-3.2 (3.3) & 2.94 & (1.6) $1.7-2.2$ & 2.00 & (1.2) $1.3-1.7(1.8)$ & 1.47 & 30 \\
\hline Vlasák 1808/39 & (2.3) 2.6-3.2 (3.3) & 2.87 & (1.7) $1.8-2.2(2.3)$ & 1.98 & (1.3) $1.4-1.6(1.7)$ & 1.46 & 30 \\
\hline Sertulicium granuliferum & (4.8) 4.9-7.3 (8.0) & 5.75 & (2.3) 2.5-3.3 (3.5) & 2.90 & (1.5) 1.6-2.4 (2.5) & 2.02 & 270 \\
\hline Kotiranta 26776 & $5.1-6.7(6.8)$ & 5.70 & (2.6) 2.7-3.2 (3.3) & 2.88 & (1.6) $1.8-2.2(2.3)$ & 1.98 & 30 \\
\hline Larsson 12282 & (5.0) 5.1-6.0 (6.1) & 5.46 & (2.7) $2.8-3.2(3.3)$ & 3.02 & (1.6) $1.7-2.0(2.1)$ & 1.81 & 30 \\
\hline LE 299057 & (4.8) 4.9-6.0 (6.1) & 5.40 & (2.5) $2.6-3.2$ & 2.83 & (1.6) 1.7-2.1 (2.2) & 1.91 & 30 \\
\hline Miettinen 16083 & (6.0) 6.1-7.3 (8.0) & 6.76 & (2.7) $2.8-3.3(3.5)$ & 3.07 & (1.8) $2.0-2.4$ & 2.21 & 30 \\
\hline Spirin 3503 & (5.1) 5.2-6.2 (6.3) & 5.82 & (2.6) $2.7-3.1(3.2)$ & 2.85 & (1.8) 1.9-2.3 (2.4) & 2.04 & 30 \\
\hline Spirin 9296 & $5.2-6.5(7.0)$ & 5.95 & (2.6) $2.7-3.2$ & 2.87 & (1.6) $1.8-2.4(2.5)$ & 2.41 & 30 \\
\hline Spirin 9898 & (4.9) 5.0-6.1 (6.2) & 5.46 & (2.6) $2.7-3.2(3.3)$ & 2.86 & (1.6) $1.7-2.1(2.2)$ & 1.92 & 30 \\
\hline Spirin 9977 & (4.8) 5.1-6.6 (7.2) & 5.63 & (2.6) $2.7-3.3(3.5)$ & 2.97 & (1.5) $1.6-2.2(2.3)$ & 1.91 & 30 \\
\hline Telleria 16566 & (4.9) 5.0-6.1 (6.2) & 5.56 & (2.3) $2.5-3.1(3.3)$ & 2.78 & (1.7) $1.8-2.2(2.3)$ & 2.00 & 30 \\
\hline S. jacksonii & (5.1) 5.2-8.2 (8.6) & 6.60 & (2.7) $2.8-4.1(4.2)$ & 3.29 & (1.5) 1.6-2.6 (2.7) & 2.02 & 170 \\
\hline holotype & (6.1) 6.2-8.0 (8.2) & 7.13 & (2.7) $2.8-3.2(3.3)$ & 2.99 & $2.2-2.6(2.7)$ & 2.39 & 20 \\
\hline Bernicchia 5464 & (5.3) $5.7-7.3$ (7.8) & 6.52 & (3.2) $3.3-4.1(4.2)$ & 3.66 & (1.5) 1.6-2.1 (2.2) & 1.79 & 30 \\
\hline Laurila 3256b & (5.2) $5.3-7.2$ & 6.43 & (2.7) $2.8-3.8(4.0)$ & 3.23 & (1.8) $1.9-2.5$ (2.6) & 2.00 & 30 \\
\hline Miettinen 17141.2 & (6.0) 6.1-8.2 (8.6) & 6.96 & $3.0-3.4(3.5)$ & 3.21 & (1.9) $2.0-2.5$ & 2.17 & 30 \\
\hline Spirin 10425 & (5.2) 5.6-7.4 (8.0) & 6.49 & (2.8) 2.9-3.8 (3.9) & 3.30 & (1.6) $1.7-2.3$ (2.6) & 1.97 & 30 \\
\hline Svantesson 699 & (5.1) 5.2-6.8 (7.1) & 6.08 & (3.0) 3.1-3.9 (4.0) & 3.36 & (1.5) $1.6-1.9$ (2.3) & 1.81 & 30 \\
\hline S. lateclavigerum & (4.3) 4.6-7.2 (7.3) & 5.57 & (2.1) 2.2-3.2 & 2.71 & (1.7) 1.8-2.4 (2.5) & 2.07 & 60 \\
\hline holotype & (4.9) 5.0-7.2 (7.3) & 5.82 & (2.5) $2.6-3.2$ & 2.84 & (1.7) $1.8-2.3$ (2.4) & 2.05 & 30 \\
\hline Spirin 13457 & (4.3) 4.6-6.2 (7.2) & 5.32 & (2.1) $2.2-3.0(3.1)$ & 2.57 & (1.7) $1.8-2.4(2.5)$ & 2.09 & 30 \\
\hline S. niveocremeum & (5.2) 5.5-8.6 (9.8) & 6.86 & (2.6) $2.7-4.2(4.3)$ & 3.25 & (1.6) 1.7-2.4 (2.6) & 2.12 & 120 \\
\hline holotype & (5.2) $5.5-7.8(9.8)$ & 6.62 & (2.6) $2.7-3.6(4.3)$ & 3.12 & (1.7) $1.9-2.4(2.5)$ & 2.12 & 30 \\
\hline Kotiranta 26267 & (5.7) 6.0-7.4 (7.6) & 6.55 & (2.7) $2.8-3.4(3.5)$ & 3.08 & (1.9) $2.0-2.3(2.5)$ & 2.13 & 30 \\
\hline Miettinen 14925.3 & $(6.0) 6.1-8.6(9.2)$ & 7.46 & (3.0) 3.1-3.6 (3.8) & 3.33 & (1.7) $1.8-2.4(2.6)$ & 2.24 & 30 \\
\hline Söderholm 4050 & (6.0) 6.1-7.7 (7.8) & 6.79 & (3.0) $3.1-4.2(4.3)$ & 3.46 & (1.6) $1.7-2.2(2.3)$ & 1.97 & 30 \\
\hline S. vernale & (5.7) 5.8-8.2 (8.3) & 6.96 & (2.8) 2.9-3.8 (3.9) & 3.30 & (1.6) $1.8-2.5(2.9)$ & 2.12 & 60 \\
\hline holotype & (5.7) 6.1-8.2 (8.3) & 7.02 & (2.8) $2.9-3.8$ & 3.28 & (1.6) $1.8-2.5$ (2.9) & 2.15 & 30 \\
\hline Spirin 14244 & (5.7) 5.8-8.0 (8.1) & 6.90 & (2.9) 3.0-3.8 (3.9) & 3.31 & (1.6) $1.8-2.3(2.4)$ & 2.09 & 30 \\
\hline Sistotremastrum aculeatum & (4.1) 4.2-6.2 (6.8) & 5.03 & (2.1) 2.2-3.1 (3.3) & 2.58 & (1.5) 1.6-2.4 (2.7) & 1.97 & 120 \\
\hline holotype & (4.1) 4.2-6.2 (6.8) & 5.07 & (2.2) $2.3-3.1(3.3)$ & 2.65 & $(1.5-) 1.6-2.4(2.7)$ & 1.94 & 60 \\
\hline Dunaev KUN1105d & $4.2-5.3(5.4)$ & 4.98 & (2.1) $2.2-3.0(3.1)$ & 2.60 & $(1.6-) 1.7-2.4(2.5)$ & 1.94 & 30 \\
\hline Miettinen 10380.1 & (4.2) $4.3-6.1(6.2)$ & 5.05 & (2.2) $2.3-2.8(3.0)$ & 2.50 & $1.8-2.2(2.3)$ & 2.03 & 30 \\
\hline S. aculeocrepitans & (4.0) $4.1-5.6(5.8)$ & 4.77 & (2.1) 2.2-2.8 (2.9) & 2.33 & $1.8-2.5(2.6)$ & 2.06 & 60 \\
\hline Larsson 16097 & (4.4) $4.5-5.6(5.8)$ & 5.10 & (2.1) $2.2-2.8(2.9)$ & 2.35 & (1.8) $1.9-2.5$ (2.6) & 2.19 & 30 \\
\hline Larsson 16478 & (4.0) 4.1-5.0 (5.1) & 4.44 & (2.1) $2.2-2.6(2.7)$ & 2.30 & $1.8-2.1(2.2)$ & 1.93 & 30 \\
\hline S. confusum & (5.3) 5.7-7.6 (7.9) & 6.56 & $2.9-3.9(4.0)$ & 3.24 & (1.7) $1.8-2.3(2.6)$ & 2.03 & 60 \\
\hline holotype & (5.3) 5.7-7.3 (7.8) & 6.54 & $2.9-3.4(3.8)$ & 3.16 & (1.7) $1.8-2.3$ (2.6) & 2.07 & 30 \\
\hline Larsson 16004 & (5.3) 5.7-7.6 (7.9) & 6.57 & $3.0-3.9(4.0)$ & 3.32 & (1.7) $1.8-2.3(2.4)$ & 1.99 & 30 \\
\hline S. denticulatum & (3.9) $4.0-5.3(5.4)$ & 4.51 & (2.0) 2.1-3.0 (3.1) & 2.48 & (1.5) $1.6-2.3(2.4)$ & 1.83 & 30 \\
\hline holotype & (3.9) $4.0-5.3$ (5.4) & 4.51 & (2.0) $2.1-3.0(3.1)$ & 2.48 & (1.5) $1.6-2.3(2.4)$ & 1.83 & 30 \\
\hline S. fibrillosum & (4.0) 4.2-5.2 (5.6) & 4.72 & (2.1) 2.2-2.9 (3.0) & 2.53 & $(1.6-) 1.7-2.1(2.2)$ & 1.88 & 20 \\
\hline Larsson 16988 & (4.0) 4.2-5.2 (5.6) & 4.72 & (2.1) $2.2-2.9(3.0)$ & 2.53 & $(1.6-) 1.7-2.1(2.2)$ & 1.88 & 20 \\
\hline S. geminum & (3.8) 3.9-5.2 (5.5) & 4.28 & (1.9) $2.0-2.6(2.9)$ & 2.24 & (1.7) $1.8-2.2(2.3)$ & 1.92 & 30 \\
\hline holotype & (3.8) 3.9-5.2 (5.5) & 4.28 & (1.9) $2.0-2.6(2.9)$ & 2.24 & (1.7) $1.8-2.2(2.3)$ & 1.92 & 30 \\
\hline S. induratum & (4.0) $4.1-5.3(5.8)$ & 4.55 & (2.5) 2.6-3.1 (3.2) & 2.83 & 1.4-1.8 (1.9) & 1.62 & 60 \\
\hline
\end{tabular}


Table 2 (continued)

\begin{tabular}{|c|c|c|c|c|c|c|c|}
\hline Species/specimen & $\mathrm{L}^{\prime}$ & $\mathrm{L}$ & $\mathrm{W}^{\prime}$ & W & $\mathrm{Q}^{\prime}$ & Q & $\mathrm{n}$ \\
\hline holotype & (4.1) $4.2-5.3(5.8)$ & 4.73 & (2.5) 2.6-3.0 & 2.79 & (1.5) 1.6-1.8 (1.9) & 1.70 & 30 \\
\hline Spirin 8804 & (4.0) 4.1-5.0 (5.1) & 4.37 & (2.5) 2.6-3.1 (3.2) & 2.86 & $1.4-1.8(1.9)$ & 1.53 & 30 \\
\hline S. mendax & (4.0) $4.2-7.0$ (7.2) & 5.27 & (2.4) $2.5-3.5$ (3.6) & 2.93 & (1.3) 1.4-2.1 (2.3) & 1.80 & 180 \\
\hline holotype & (4.7) $4.8-6.2$ & 5.60 & (2.7) $2.9-3.5$ (3.6) & 3.09 & (1.6) $1.7-2.1(2.3)$ & 1.82 & 30 \\
\hline Larsson 12022 & (4.6) $4.8-5.6$ & 5.12 & (2.6) $2.7-3.0(3.1)$ & 2.85 & (1.6) $1.7-1.9$ & 1.80 & 30 \\
\hline Nordén 9579 & (4.7) $4.8-5.8(6.0)$ & 5.33 & (2.6) $2.7-3.1(3.2)$ & 2.93 & (1.6) $1.7-2.0(2.1)$ & 1.82 & 30 \\
\hline Spirin 10060 & (5.0) 5.1-7.0 (7.2) & 5.71 & (2.6) $2.7-3.2(3.3)$ & 2.93 & $1.7-2.3(2.4)$ & 1.95 & 30 \\
\hline Spirin 10107 & (4.2) $4.3-5.9(6.1)$ & 5.11 & (2.7) $2.8-3.2(3.5)$ & 3.00 & (1.3) $1.4-1.9$ (2.0) & 1.71 & 30 \\
\hline Vlasák 1110/14.1 & (4.0) 4.2-5.4 (5.5) & 4.75 & (2.4) $2.5-3.0$ & 2.76 & (1.4) $1.5-1.9$ & 1.72 & 30 \\
\hline$S$. rigidum & (3.8) 4.0-5.1 (5.2) & 4.44 & $2.0-2.5(2.7)$ & 2.22 & (1.6) 1.7-2.2 (2.3) & 2.01 & 30 \\
\hline holotype & (3.8) 4.0-5.1 (5.2) & 4.44 & $2.0-2.5(2.7)$ & 2.22 & (1.6) $1.7-2.2(2.3)$ & 2.01 & 30 \\
\hline S. suecicum & (4.1) 4.2-6.2 (6.3) & 4.90 & (2.1) 2.2-3.1 (3.2) & 2.53 & (1.5) 1.6-2.4 (2.5) & 1.93 & 180 \\
\hline holotype & (4.8) 5.1-6.2 (6.3) & 5.55 & (2.4) $2.5-3.1(3.2)$ & 2.80 & (1.7) $1.8-2.2(2.3)$ & 1.99 & 30 \\
\hline Kunttu 5959 & (4.1) $4.2-5.2$ & 4.70 & $2.2-2.8(2.9)$ & 2.52 & (1.6) $1.7-2.1(2.2)$ & 1.88 & 30 \\
\hline Larsson 11849 & (4.1) 4.2-5.7 (5.8) & 4.91 & (2.1) $2.2-2.9$ & 2.42 & (1.7) $1.8-2.4(2.5)$ & 2.05 & 30 \\
\hline Miettinen 11044 & (4.5) 4.6-5.9 (6.1) & 5.12 & $2.2-2.9(3.0)$ & 2.50 & (1.7) $1.8-2.3(2.5)$ & 2.06 & 30 \\
\hline Miettinen 14550.1 & (4.1) 4.2-5.1 (5.3) & 4.60 & $2.2-2.9(3.0)$ & 2.52 & (1.5) 1.6-2.0 (2.1) & 1.83 & 30 \\
\hline Spirin 8932 & (4.1) $4.2-5.3(5.8)$ & 4.56 & (2.1) $2.2-2.9$ (3.0) & 2.42 & (1.7) $1.8-2.1(2.2)$ & 1.89 & 30 \\
\hline S. vigilans & (6.1) 6.3-11.2 (11.4) & 8.68 & (3.0) 3.1-5.4 (6.2) & 4.35 & (1.6) $1.7-2.5$ (2.6) & 2.01 & 150 \\
\hline holotype & (7.0) 7.2-9.1 (9.4) & 8.09 & (3.9) 4.0-5.2(5.8) & 4.49 & (1.6) $1.7-2.0(2.1)$ & 1.81 & 30 \\
\hline Eriksson 9106 & (8.0) 8.3-10.5 (10.6) & 9.34 & (3.8) 4.0-5.0 (5.1) & 4.44 & (1.8) 1.9-2.2 (2.3) & 2.11 & 30 \\
\hline Fonneland 2011-77 & (8.1) 8.8-11.2 (11.4) & 9.95 & (4.0) 4.1-5.1 (5.2) & 4.50 & (1.8) 1.9-2.5 (2.6) & 2.22 & 30 \\
\hline Häyrén 1934 & (7.7) $7.8-10.3(10.9)$ & 8.61 & (4.1) $4.2-5.4(6.2)$ & 4.69 & (1.7) $1.8-2.0(2.1)$ & 1.84 & 30 \\
\hline Spirin 8778 & (6.1) 6.3-8.8 (8.9) & 7.43 & (3.0) $3.1-4.3(4.5)$ & 3.64 & (1.7) $1.8-2.3$ (2.4) & 2.05 & 30 \\
\hline
\end{tabular}

Dist., Mokhovoe, Q. robur, 24.VII.2011 Volobuev (LE 314057*); Sverdlovsky Dist., Maryevka, Betula pendula, 1.IX.2012 Volobuev (LE 314055*); Uritsky Dist., Naryshkino, B. pendula, 6.X.2012 Volobuev (LE 292194*), Q. robur, 25.VI.2011 Volobuev (LE 314054*), 6.X.2012 Volobuev (LE 314053*). Primorie Reg.: Ternei Dist., Maisa, hardwood stump, 9.IX.1990 Larsson 7182 (GB), Alnus sp. (?), 12.IX.1990 Larsson 7447 (GB), Blagodatnoe, Alnus sp., 23.IX.1990 Larsson 8140 (GB). Tuva: Turgen, Larix sibirica (charred corticated log), 23.VIII.2014 Kotiranta 26776* (H). Spain. Burgos: Ordun, Fagus sylvatica, 12.XI.1977 Ryvarden 15329 (O, H). Santander: Cavadonga, Sambucus, 14.IX.1977 Ryvarden 15365 (O, H). Ukraine. Donetsk Reg.: Slovyansk Dist., Pyskunkivka, Salix alba, 20.XI.2010 Akulov (CWU 4704*). USA. Massachusetts: Hampshire Co., South Hadley, decayed wood, 4.V.2013 Miettinen 16083* (H); Worcester Co., Holden, cut bole, 26.IX.2011 Miettinen 14813.2* (H), Worcester, ?Acer saccharum, 8.IX.2013 Miettinen $16834(\mathrm{H})$, Populus tremuloides, 5.X.2013 Miettinen $17267.4(\mathrm{H})$, Quercus sp., 6.X.2013 Miettinen 17272* (H), Acer sp., 26.X.2014 Miettinen 19035 (H). Michigan: Barry Co., Yankee Springs, Quercus sp., 3.IX.1955 Cain (H ex TRTC 31857). Tennessee: Cocke Co., Cosby, decayed wood, 13.VII.2004 K.H. Larsson
12198 (GB), 14.VII.2004 K.H. Larsson 12216 (GB), 17.VII.2004 K.H. Larsson 12282*, 12297* (GB).

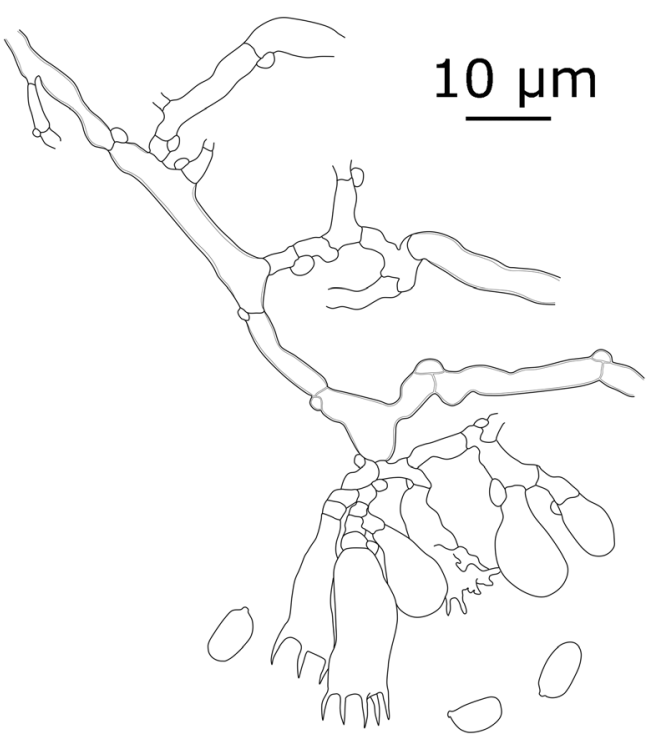

Fig. 7 Microscopic structures of S. jacksonii (Svantesson 699): hyphae, hymenial cells, and basidiospores 
Sertulicium jacksonii (Liberta) Spirin \& K.H. Larss., comb. nov.-Figs. 7 and 10

\section{MB 833944}

三Paullicorticium jacksonii Liberta, Brittonia 14: 223, 1962. Holotype. Canada. Ontario: York, Maple, decayed wood, 11.IX.1943 Jackson (TRTC 18722, studied).

Basidiocarps effused, pruinose, hardly visible by the naked eye, $0.02-0.03 \mathrm{~mm}$ thick. Hymenial surface whitish, smooth or porulose. Margin indistinct.

Hyphal structure monomitic; hyphae clamped. Subicular hyphae sparse, randomly arranged, slightly to moderately thick-walled, branched mostly at sharp angles, often anastomosing, (2.2-) 2.3-6.4 (-6.8) $\mu \mathrm{m}$ in diam. $(\mathrm{n}=68 / 5)$. Subhymenial hyphae thin- or slightly thick-walled, densely and randomly arranged, (2.0-) 2.1-3.8 (-4.0) $\mu \mathrm{m}$ in diam. ( $\mathrm{n}=100 / 5$ ). Hyphidia occasionally present, simple, slightly projecting, 3-4 $\mu \mathrm{m}$ in diam. Basidia clavate, 4-6-spored, gradually tapering to the basal part, (11.6-) 12.2-23.8 ($24.7) \times(5.8-) 5.9-9.3(-9.4) \mu \mathrm{m}(\mathrm{n}=72 / 4)$, a few pleural basidia seen. Basidioles broadly clavate to subglobose, (8.2-) 8.3-13.2 (-13.3) × (4.7-) 5.7-9.2 (-9.3) $\mu \mathrm{m}(\mathrm{n}=35 /$ 4). Basidiospores cylindrical to narrowly ellipsoid, straight or slightly curved, longest spores slightly tapering to the distal end, (5.1-) 5.2-8.2 (-8.6) × (2.7-) 2.8-4.1 (-4.2) $\mu \mathrm{m}(\mathrm{n}=$ $170 / 6), \mathrm{L}=6.08-7.13, \mathrm{~W}=2.99-3.66, \mathrm{Q}=1.79-2.39$.

Remarks. Paullicorticium jacksonii was described from Canada but later rarely treated as a species of its own. Eriksson et al. (1978) placed P. jacksonii among the synonyms of Sistotremastrum niveocremeum, and the species has been interpreted that way up to the present moment. Our data confirm that $P$. jacksonii is a good species belonging to Sertulicium. In addition to the type collection, we studied specimens from the USA and Europe.

Sertulicium jacksonii belongs to a difficult complex of extremely thin, conifer-dwelling species hardly distinguishable via morphological traits. Among them, S. jacksonii can be recognized by its wide subicular hyphae with variably thickened walls. Two similar-looking species, Sertulicium vernale and S. lateclavigerum, have narrower subicular hyphae than those of $S$. jacksonii. Additionally, $S$. lateclavigerum possesses narrower basidiospores than in the other two species (Table 2). However, the differences listed here were detected from a very restricted set of specimens. Further studies with broader sampling may improve our observations considerably. Paullicorticium ansatum Liberta, yet another morphologically similar although phylogenetically unrelated species, may be easily separated from Sertulicium spp. due to ansiform (loop-like) clamps and basidia with up to eight sterigmata.

Specimens examined. Italy. Trentino-Alto Adige: Bolzano, Picea abies, 30.VIII.1990 Bernicchia 5464 (O ex HUBO). Norway. Oppland: Jevnaker, P. abies, 4.X.2011 Svantesson 699*(O). Russia. Karelia:
Medvezhiegorsk Dist., Krivozero, P. abies, 19.VI.1942 Laurila $3256 b(\mathrm{H})$. Leningrad Reg.: Boksitogorsk Dist., Kolp', P. abies, 28.VII.2016 Spirin 10425*(H). USA. New York: Essex Co., Arbutus Lake, Abies sp., 22.IX.2013 Miettinen $17141 *(\mathrm{H})$.

Sertulicium lateclavigerum (Boidin \& Gilles) Spirin \& Viner, comb. nov.-Fig. 8

MB 833945

$\equiv$ Sistotremastrum lateclavigerum Boidin \& Gilles, Bull. Soc. Mycol. France 110: 217, 1994. Holotype. France. Landes: Tartas, Pinus pinaster (fallen branch), 17.VI.1988 Gilles $765 *$ (LY-JB 13467, studied).

Basidiocarps effused, pruinose, rather loose or compact, covering a few $\mathrm{cm}, 0.02-0.05 \mathrm{~mm}$ thick. Hymenial surface cream-colored, smooth or porulose. Margin indistinct.

Hyphal structure monomitic; hyphae clamped. Subicular hyphae sparse, randomly arranged, thin- or only slightly thick-walled, branched mostly at sharp angles, often anastomosing, (2.2-) 2.3-4.3 (-4.9) $\mu \mathrm{m}$ in diam. $(\mathrm{n}=40 / 2)$. Subhymenial hyphae thin-walled (wall distinct), rather loosely and randomly arranged, (2.0-) 2.1-3.7 (-2.8) $\mu \mathrm{m}$ in diam. (n $=40 / 2$ ). Cystidia occasionally present, thin-walled, easily collapsing, broadly clavate or subglobose, $19-33 \times 9-16.5 \mu \mathrm{m}$. Hyphidia occasionally present, embedded, simple, 2-2.5 $\mu \mathrm{m}$ in diam. Basidia clavate, 4-6-spored, gradually tapering to the basal part, (9.3-) 9.9-20.7 (-21.2) × (4.4-) 4.8-9.3 (-11.1) $\mu \mathrm{m}(\mathrm{n}=40 / 2)$, a few pleural basidia seen. Basidioles broadly clavate to bladder-shaped or subglobose, (7.3-) 7.9-10.2 ($10.3) \times(4.4-)$ 5.4-6.6 (-7.9) $\mu \mathrm{m}(\mathrm{n}=20 / 1)$. Basidiospores cylindrical to subfusiform, sometimes slightly curved, (4.3-)

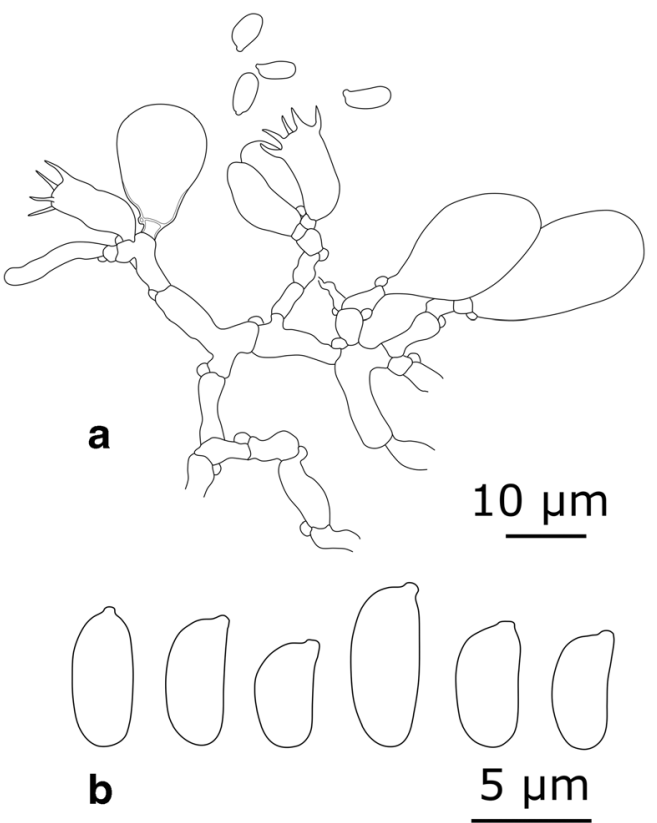

Fig. 8 Microscopic structures of S. lateclavigerum (holotype). a Hyphae and hymenial cells. b Basidiospores 
4.6-7.2 (-7.3) $\times(2.1-) 2.2-3.2 \mu \mathrm{m}(\mathrm{n}=60 / 2), \mathrm{L}=5.32-5.82$, $\mathrm{W}=2.57-2.84, \mathrm{Q}=2.05-2.09$.

Remarks. Sistotremastrum lateclavigerum was described based on a single collection from France. The diagnostic features treated in the protologue were the bubble-like cystidia, basidia with up to 8 sterigmata and rather narrow and slightly curved basidiospores (Boidin and Gilles 1994). Sequences obtained from the holotype were published by Gruhn et al. (2018). They indicate that the species belongs to Sertulicium and it is not closely related to the similarly looking $S$. jacksonii and $S$. vernale. Morphological differences of $S$. lateclavigerum versus the two latter species are presented under S. jacksonii. Paullicorticium allantosporum J. Erikss. has basidiospores similar to $S$. lateclavigerum, (5.2-) 5.3-7.2 $\times(2.3-) 2.4-3.2(-3.3) \mu \mathrm{m}(\mathrm{n}=30 / 1), \mathrm{L}=6.10, \mathrm{~W}=2.79, \mathrm{Q}=$ 2.20 , but they are more clearly curved and have large oil droplets in the cytoplasm. Moreover, basidia of $P$. allantosporum are 6-8-sterigmatic, with a clearly contracted and more or less sinuous basal part.

We studied the holotype of S. lateclavigerum and observed basidia with 4-6 sterigmata only. In our opinion, the presence of globose or broadly clavate cystidia-like cells is insufficient as a single diagnostic character in Sistotremastrum s. 1. We observed hymenial cells of the same shape in one collection of S. jacksonii (Laurila 3256b), in S. niveocremeum (Van Autgaerden $S-20$ ), as well as in three representatives of Sistotremastrum s. str. (holotype of S. geminum and two senescent collections of $S$. mendax and $S$. vigilans, respectively). On the other hand, another collection of $S$. lateclavigerum studied by us (Spirin 13457) is totally devoid of cystidia-like cells.

So far, S. lateclavigerum has been collected in France and Slovenia. However, environmental sequences in GenBank and UNITE point to a broader distribution area of this species. One of them came from Vietnam (MF942562) while the second (UDB051695) is from Estonia.

Specimens examined. Slovenia. Gorenjska: Mojstrana, Triglavska Bistrica, P. abies, 28.IX.2019 Spirin 13457* (H).

Sertulicium niveocremeum (Höhn. \& Litsch.) Spirin \& K.H. Larss., comb. nov.-Fig. 10

MB 833946

$\equiv$ Corticium niveocremeum Höhn. \& Litsch., Sitzungsb. Kaiserl. Akad. Naturwiss. Mat.-Naturwiss. Klasse I 117:1117, 1908. Holotype. Austria. Niederösterreich: WienUmgebung, Unter-Tullnerbach, Fagus sylvatica, 11.III.1905 Höhnel (W 16251, studied).

= Galzinia vesana Boidin \& Gilles, Bull. Soc. Mycol. France 106: 158, 1990. Holotype. France. Landes: St. Perdon, Quercus pedunculata, 2.III.1989 Gilles 828 (LY-JB 13675, studied).

Basidiocarps effused, initially pruinose, later compact, covering several $\mathrm{cm}, 0.03-0.1 \mathrm{~mm}$ thick. Hymenial surface whitish to cream-colored or pale ochraceous, smooth or porulose. Margin first pruinose, later compact and sharply delimited.

Hyphal structure monomitic; hyphae clamped. Subicular hyphae interwoven to subparallel, slightly thick-walled, branched mostly at right angles, (3.6-) 3.7-5.3 (-5.6) $\mu \mathrm{m}$ in diam. $(n=40 / 2)$; some hyphae inflated at septa up to $6 \mu \mathrm{m}$ in diam. Subhymenial hyphae thin- or slightly thick-walled, densely arranged, some short-celled and slightly inflated, (2.2-) 2.3-4.3 (-4.5) $\mu \mathrm{m}$ in diam. $(\mathrm{n}=80 / 4)$. Hyphidia occasionally present, embedded, simple, $2.5-4.5 \mu \mathrm{m}$ in diam. Basidia clavate, 4-6-spored, gradually tapering to the basal part, (10.7-) 10.8-24.3 (-26.7) × (5.1-) 5.2-8.3 (-8.4) $\mu \mathrm{m}$ $(n=60 / 3)$, some basidia slightly thick-walled at the basal part. Basidioles broadly clavate to bladder-shaped or subglobose, (7.2-) 8.4-14.3 (-14.7) × (4.1-) 4.2-8.3 (-8.4) $\mu \mathrm{m}(\mathrm{n}=80 / 4)$. Basidiospores cylindrical, straight or slightly curved, (5.2-) $5.5-8.6(-9.8) \times(2.6-) 2.7-4.2(-4.3) \mu \mathrm{m}(\mathrm{n}=120 / 4), \mathrm{L}=$ $6.55-7.46, \mathrm{~W}=3.08-3.46, \mathrm{Q}=1.97-2.24$.

Remarks. Sertulicium niveocremeum is distributed in temperate-boreal Eurasia. It inhabits angiosperm hosts, mainly still attached or recently fallen branches but it may occur also on rather rotten wood. For a long time, $S$. niveocremeum has been mixed up with another angiosperm-dwelling species, $S$. granuliferum; their differences are discussed under the latter species. We studied the type material and a recent collection of Galzinia vesana from France; in our opinion, it is merely a monstrous form of $S$. niveocremeum with unusually long basidia and basidiospores, as well as occasionally branched sterigmata. DNA data from recently collected specimens confirmed this synonymy. While introducing G. vesana, Boidin and Gilles (1990) referred to a description and illustration of Galzinia sp. from Sweden (Eriksson and Ryvarden 1975: 398) as another possible record of that species. We re-checked the specimen illustrated by Eriksson and concluded that it belongs to Sertulicium, being morphologically most similar to S. niveocremeum. However, its exact identity is still uncertain, and it is therefore treated as Sertulicium sp. 2 under Specimens examined.

Some specimens of $S$. niveocremeum collected in the winter or spring are dominated by four-sterigmatic basidia. Only a few 5-6-sterigmatic basidia were detected in such specimens after meticulous study. Moreover, these collections usually have longer basidiospores than in most other specimens of S. niveocremeum (in particular, reaching $14.5 \mu \mathrm{m}$ long in specimen Van Autgaerden S-20) and thus can be mixed up with Sistotremastrum vigilans. The latter species, introduced below, has constantly four-sterigmatic basidia and it occurs exclusively on coniferous hosts while $S$. niveocremeum seems to be restricted to angiosperms.

Specimens examined. Belgium. Antwerpen: Zoersel, Zoerselbos, deciduous wood, 16.V.2019 Van Autgaerden 
$S-20^{*}$ (GENT). Oost-Vlaanderen: Kluisbergen, Kluisbos, deciduous wood, 20.II.2017 Schoutteten 17-001 (GENT), 13.III.2017 Schoutteten 17-202 (GENT). Finland. VarsinaisSuomi: Lohja, Tamminiemi, Corylus avellana, 5.XI.2020 Spirin 14241 (H). Uusimaa: Helsinki, Veräjämäki, Sorbus aucuparia (?), 19.X.2011 Miettinen 14925.3* (H). EteläHäme: Kangasala, Lorunkorpi, Salix sp., 23.III.2009 Söderholm 4050* (H). France. Haut-Rhin: Sainte-Marieaux-Mines, Freland, Le Limbach, F. excelsior, 12.X.2019 Spirin $13566(\mathrm{H})$. Pyrenées-Orientales: Argelès-sur-Mer, angiosperm (twigs), 2.XI.2008 K.H. Larsson 13727* (GB). Italy. Emilia-Romagna: Bologna, Quercus sp., 3.XI.1983 Bernicchia 1849 (O ex HUBO), 16.X.1984 Ryvarden 22225 (O). Sardinia: Cagliari, 17.III.2003 Arras 897 (H ex HUBO); Nuoro, Quercus sp., 30.III.2010 Bernicchia 8593 (O ex HUBO). Russia. Krasnoyarsk Reg.: Turukhansk Dist., Bor, Alnus sibirica, 16.VIII.2013 Kotiranta 26267* (H). Sakha Rep.: Nizhnekolymsk Dist., Tsherski, Alnus fruticosa, 18.VIII.1972 Parmasto (H ex TAAM 56632). Spain. Canary Islands: Tenerife, Las Mercedes, decayed wood, 18.I.1974 Ryvarden 12639 (O, H). United Kingdom. England: Hampshire, New Forest Nat. Park, F. sylvatica, 5.II.1994 Legon (GB).

Sertulicium vernale Spirin \& Volobuev, sp. nov.-Fig. 9 MB 833947

Holotype. Finland. Etelä-Häme: Kangasala, Pikku-Salmus, $61.4138^{\circ} 23,9275^{\circ} \pm 300 \mathrm{~m}$, moist depression in rather old Picea forest, on a fallen, decorticated Picea abies, 10.V.2007 Söderholm 3886* (H 6003442, isotype-LE).

Etymology. Vernalis (Lat., adj.) - vernal, occurring in the spring.

Basidiocarps effused, pruinose, rather loose, covering a few $\mathrm{cm}, 0.02-0.04 \mathrm{~mm}$ thick. Hymenial surface greyish, smooth or porulose. Margin indistinct.

Hyphal structure monomitic; hyphae clamped. Subicular hyphae sparse, randomly arranged, slightly to moderately thick-walled, branched mostly at sharp angles, often anastomosing, (2.2-) 2.3-4.8 (-5.2) $\mu \mathrm{m}$ in diam. $(\mathrm{n}=40 / 2)$. Subhymenial hyphae thin-walled (wall usually distinct), rather tightly and randomly arranged, (2.0-) $2.1-4.1(-4.2) \mu \mathrm{m}$ in diam. $(n=40 / 2)$, sometimes bearing bubble-like cells, 10-12 $\times 8-11 \mu \mathrm{m}$. Hyphidia rare, embedded, simple, $2.5-3.5 \mu \mathrm{m}$ in diam. Basidia clavate, 4-6-spored, gradually tapering to the basal part, (11.2-) 11.8-27.4 (-37.2) × (5.0-) 5.1-7.2 (-7.3) $\mu \mathrm{m}(\mathrm{n}=44 / 2)$. Basidioles broadly clavate to bladder-shaped or subglobose, (6.0-) 6.9-10.2 (-10.8) × (4.4-) 5.2-6.8 (-6.9) $\mu \mathrm{m}(\mathrm{n}=20 / 1)$. Basidiospores narrowly ellipsoid to broadly cylindrical, sometimes slightly curved, (5.7-) 5.8-8.2 (-8.3) $\times$ (2.8-) 2.9-3.8 (-3.9) $\mu \mathrm{m}(\mathrm{n}=60 / 2), \mathrm{L}=6.90-7.02, \mathrm{~W}=$ $3.28-3.31, \mathrm{Q}=2.09-2.15$.

Remarks. Sertulicium vernale is morphologically very similar to $S$. jacksonii, and it can be differentiated from the latter

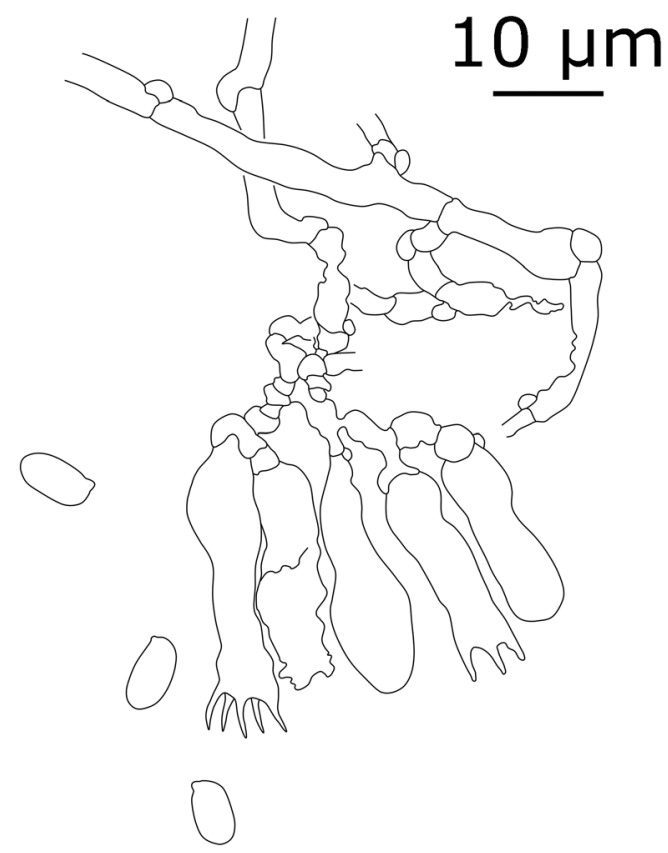

Fig. 9 Microscopic structures of S. vernale (holotype): hyphae, hymenial cells, and basidiospores

species by its narrower subicular hyphae and longer basidia. The species is so far known from two localities in Finland and two environmental sequence from Sweden (AY805625Menkis et al. 2004) and CO, USA (JX136434-Huffman et al. 2013). The North American sequence is slightly different ( $2 \mathrm{bp}$ ) from the two European ones, and it may still belong to an undescribed sibling species.

Specimens examined. Finland. Varsinais-Suomi: Lohja, Tamminiemi, P. abies, 5.XI.2020 Spirin 14244 (H).

Sistotremastrum J. Erikss., Symb. Bot. Upsalienses 16 (1): 62, 1958.

Basidiocarps effused, thin to rather substantial (up to $0.2 \mathrm{~mm}$ thick), pruinose to waxy-compact. Hymenophore smooth, warted or odontioid-semiporoid. Hyphal structure monomitic; all hyphae clamped, CB (+), easily collapsing, in odontioid species with a more or less clear difference between trama and subhymenium. Cystidia absent in all but one species, hyphidia present, simple or accidentally branched. Basidia clavate or suburniform, with 2-4 or 4-6 sterigmata, usually collapsing at the apex, senescent basidia often slightly thick-walled at the base. Basidiospores thin-walled (but the wall distinct), narrowly ellipsoid or ovoid to cylindrical, inamyloid, acyanophilous, contents homogeneous and $\mathrm{CB}$ $(+)$. On rotten wood of deciduous trees and conifers.

Generic type. Sistotremastrum suecicum Litsch. ex J. Erikss.

The genus is redefined here to exclude members of the Sistotremastrum niveocremeum complex subsumed under Sertulicium. Differences between the two genera are discussed above. Extending Sistotremastrum s. str. with a number of four- 
sterigmatic taxa makes it difficult to delimit the genus from Brevicellicium and from smooth-spored Trechispora species. Trechispora differs by generally soft basidiocarps, short, often almost isodiametric subhymenial cells, and presence of ampullate septa on subicular hyphae. Brevicellicium species have similar subhymenial cells as in Trechispora but lack ampullate septa in the subiculum, which is poorly developed. Moreover, basidia in the four-sterigmatic Sistotremastrum spp. are more or less clearly suburniform, i.e., slightly constricted at the middle and narrowed at the apical part (except $S$. vigilans with exceptionally long, clavate-utriform basidia) while they are barrelshaped or short-clavate in Brevicellicium and Trechispora.

All but one four-sterigmatic representatives of the genus treated below are distributed in tropical areas. However, two unnamed sequences (CMH343, GenBank KF800434; 6S1.10.S04, GenBank EF619877) originated from the eastern part of the USA (Fig. 4). This could point towards more undescribed taxa in the four-sterigmatic species complex distributed in geographic regions with a cooler climate.

Sistotremastrum aculeatum Miettinen \& Viner, sp. nov.-Fig. 10

MB 833948

Holotype. Indonesia. Sumatera Barat: Kab. Lima Puluh Kota, Gunung Sago, $-0.30585^{\circ} 100.67762^{\circ}$, alt. $1400 \mathrm{~m}$, lower montane rainforest, on a fallen log of dicot (decay stage 3/5), 25.VII.2009 Miettinen 13799.1* (ANDA-holotype, H 7009306 - isotype).

Etymology. Aculeatus (Lat., adj.)-aculeate.

Basidiocarps effused, first soft-floccose, then rather tough, covering several to tens of $\mathrm{cm}, 0.1-0.2 \mathrm{~mm}$ thick. Hymenial

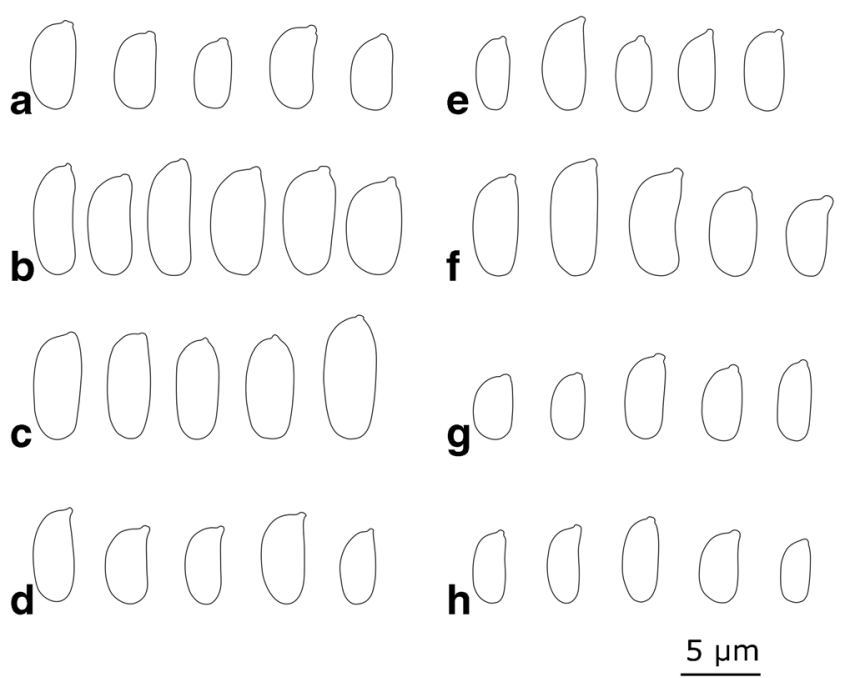

Fig. 10 Basidiospores of Sertulicium and Sistotremastrum spp. a Sertulicium granuliferum (Miettinen 14813.2). b Sertulicium jacksonii (three left-holotype, three right—Svantesson 699). c S. niveocremeum (holotype). d Sistotremastrum aculeatum (holotype). e S. aculeocrepitans (K.H. Larsson 16097). f $S$. confusum (holotype). g S. denticulatum (holotype). h S. fibrillosum (K.H. Larsson 16988) surface pale cream-colored to pale ochraceous, first warted, then odontioid, with irregularly arranged, sharp-pointed, partly fusing spines, 2-3 per $\mathrm{mm}$, up to $2 \mathrm{~mm}$ long, or semiporoid, with shallow pores $0.5-1$ per mm. Margin white to pale ochraceous, compact, in some portions with thin, white, pronounced hyphal strands. Smooth areas between spines fertile.

Hyphal structure monomitic; hyphae clamped. Subicular hyphae unevenly thick-walled, (2.8-) 2.9-5.1 (-5.2) $\mu \mathrm{m}$ in diam. $(n=40 / 2)$, often in subparallel bundles. Tramal hyphae distinctly thick-walled, rather tightly arranged, interwoven to subparallel, sometimes glued together, (2.7-) 3.0-5.6 (-6.2) $\mu \mathrm{m}$ in diam. $(\mathrm{n}=40 / 2)$. Subhymenial hyphae slightly thickwalled, short-celled, (2.3-) 2.4-4.2 (-4.6) $\mu \mathrm{m}$ in diam. $(\mathrm{n}=$ $40 / 2$ ), rarely inflated up to $6 \mu \mathrm{m}$. Rhomboid or prismatic crystals occasionally present among subicular or tramal hyphae, up to $9 \mu \mathrm{m}$ in widest dimension, solitary or in large groups. Hyphidia occasionally present, embedded or slightly projecting, simple, $2-3 \mu \mathrm{m}$ in diam. Basidia suburniform, 2 4-spored, (7.3-) 7.7-12.2 (-12.3) × (3.3-) 3.4-4.5 (-5.2) $\mu \mathrm{m}$ $(n=30 / 2)$, senescent basidia slightly thick-walled at the basal part. Basidioles clavate to bladder-shaped, 5.4-8.2 × 3.2$4.3 \mu \mathrm{m}(\mathrm{n}=10 / 1)$. Basidiospores cylindrical to narrowly ovoid, straight, (4.1-) 4.2-6.2 (-6.8) × (2.1-) 2.2-3.1 (-3.3) $\mu \mathrm{m}(\mathrm{n}=120 / 3), \mathrm{L}=4.98-5.07, \mathrm{~W}=2.50-2.65, \mathrm{Q}=1.94$ 2.03 .

Remarks. Sistotremastrum aculeatum seems to be widely distributed in South-East Asia from China to Sumatra. Morphologically, it is most similar to the South American S. aculeocrepitans. It differs from the latter species mainly by longer spines, which tend to fuse together and produce shallow, wide pores.

Specimens examined. China. Yunnan: Xishuangbanna, Mandian Waterfalls, hardwood (?), 17.VIII.2005 Miettinen 10380.1*(H). Indonesia. Riau: Indragiri Hulu, Daerah Sungai Denalo, dead polypore, 2.VII.2004 Miettinen 8829 (H). Thailand. Phang Nga: Kurabari, Kura Andaman Private Beach, hardwood, 3.II.2015 Dunaev KUN1105*(H). Singapore. Yishun, Nee Soon pipeline, hardwood, 17.III.2020 Miettinen 23533* (SING, H).

Sistotremastrum aculeocrepitans Gruhn \& Alvarado, Phytotaxa 379: 32, 2018.-Fig. 10

Basidiocarps effused, compact, covering several $\mathrm{cm}, 0.1-$ $0.2 \mathrm{~mm}$ thick. Hymenial surface cream-colored to pale ochraceous, odontioid; spines densely arranged, 3-4 per $\mathrm{mm}$, up to $0.3 \mathrm{~mm}$ long, sharp-pointed, sometimes fusing together and then fimbriate. Margin white to pale cream-colored, adnate, producing abundant thin, short, white hyphal strands. Smooth areas between spines fertile.

Hyphal structure monomitic; hyphae clamped. Subicular hyphae interwoven, distinctly thick-walled, branched mostly at sharp angles, (3.4-) 3.8-5.7 (-6.0) $\mu \mathrm{m}$ in diam. $(\mathrm{n}=20 / 1)$. Tramal hyphae distinctly thick-walled, densely arranged and 
partly glued together, (2.8-) 2.9-4.8 (-5.2) $\mu \mathrm{m}$ in diam. $(\mathrm{n}=$ $20 / 1$ ), in subhymenium more or less vertically oriented, thinor slightly thick-walled, short-celled and slightly inflated, (2.9-) 3.1-4.8 (-4.9) $\mu \mathrm{m}(\mathrm{n}=20 / 1)$. Hyphidia abundant, embedded or projecting up to $20 \mu \mathrm{m}$, simple, $2-3.5 \mu \mathrm{m}$ in diam. Basidia suburniform, 4-spored, (7.7-) 8.4-13.8 (-14.2) $\times$ (4.0-) 4.1-5.0 (-5.2) $\mu \mathrm{m}(\mathrm{n}=30 / 2)$. Basidioles clavate to bladder-shaped, 7-9 × 4-5 $\mu \mathrm{m}$. Basidiospores cylindrical to narrowly ovoid, straight, a few of the longest spores slightly lacrymoid, (4.0-) 4.1-5.6 (-5.8) × (2.1-) 2.2-2.8 (-2.9) $\mu \mathrm{m}(\mathrm{n}$ $=60 / 2), \mathrm{L}=4.44-5.10, \mathrm{~W}=2.30-2.35, \mathrm{Q}=1.93-2.19$.

Remarks. The species was introduced based on specimens from Martinique and French Guiana (Gruhn et al. 2018). We collected it in Brazil, and these specimens are the source of the present description. Differences of $S$. aculeocrepitans from $S$. denticulatum are treated under the latter species. Another similar-looking species from South America, S. fibrillosum Gruhn \& Alvarado, has soft basidiocarps and densely arranged spines covering hyphal strands, and also considerably longer basidia. Sistotremastrum aculeocrepitans is reported here as new to Brazil.

Specimens examined. Brazil. Paraíba: Areia, Reserva Mata do Pau-Ferro, angiosperm, 28.IV.2013 K.H. Larsson 16097* (URM, O). Pará: Belém, Museu Goeldi, Campus de Pesquisa, angiosperm, 25.XI.2013 K.H. Larsson 16478* (MG, O).

Sistotremastrum confusum K.H. Larss. \& Spirin, sp. nov.-Fig. 10

MB 833949

Holotype. Brazil. Pernambuco: Jaqueira, Reserva Particular do Patrimônio Natural Frei Caneca, Barragem das Moças, $-8.720420^{\circ}-35.843436^{\circ}$, on rotten angiosperm wood, 20.IV.2013 Larsson 16023* (URM, isotype-O).

Etymology. Confusus (Lat., adj.) — confusing.

Basidiocarps effused, soft, covering several cm, 0.04$0.07 \mathrm{~mm}$ thick. Hymenial surface whitish to cream-colored, smooth or irregularly tuberculate, accidentally cracking in old basidiocarps. Margin first arachnoid, with well-visible thin hyphal strands, later compact and rather clearly delimited.

Hyphal structure monomitic; hyphae clamped. Subicular hyphae interwoven to subparallel, slightly thick-walled, irregularly branched, (2.2-) 2.3-4.2 (-4.8) $\mu \mathrm{m}$ in diam. $(n=40 / 2)$. Subhymenial hyphae thin- or slightly thick-walled, rather loosely arranged, some short-celled and slightly inflated, (2.4-) 2.6-4.2 (-4.8) $\mu \mathrm{m}$ in diam. $(\mathrm{n}=40 / 2)$. Hyphidia rarely present, embedded or only slightly projecting, simple, 3$3.5 \mu \mathrm{m}$ in diam. Basidia clavate, 4-spored, gradually tapering to the basal part, (12.2-) 13.1-25.0 (-28.8) × (4.3-) 4.4-6.2 (6.3) $\mu \mathrm{m}(\mathrm{n}=22 / 2)$. Basidioles clavate to bladder-shaped, (7.3-) 7.4-11.8 (-13.0) × (4.2-) 4.3-5.3 (-6.0) $\mu \mathrm{m}(\mathrm{n}=20 /$ 2). Basidiospores cylindrical to narrowly ellipsoid or narrowly ovoid, (5.3-) 5.7-7.6 (-7.9) × 2.9-3.9 (-4.0) $\mu \mathrm{m}(\mathrm{n}=60 / 2), \mathrm{L}$
$=6.54-6.57, \mathrm{~W}=3.16-3.32, \mathrm{Q}=1.99-2.07$.

Remarks. Sistotremastrum confusum is introduced here based on two sequenced collections from Brazil. Morphologically, it is most similar to $S$. vigilans distributed in Northern Hemisphere. The latter species has considerably larger basidia and basidiospores than those of $S$. confusum.

Specimens examined. Brazil. Pernambuco: Jaqueira, Reserva Particular do Patrimônio Natural Frei Caneca, Barragem das Moças, angiosperm, 20.IV.2013 K.H. Larsson 16004* (URM, O).

Sistotremastrum denticulatum Motato-Vásquez \& Spirin, sp. nov.-Fig. 10

MB 833950

Holotype. Brazil. Saõ Paulo: Iguape, Juréia-Itatins Ecological Station, -24.417 $-47.372^{\circ}$, fallen branch, 19.II.2017 MotatoVásquez 894* (SP 467087, isotype-H 7009716).

Etymology. Denticulatus (Lat., adj.) —denticulate.

Basidiocarps effused, soft, waxy covering several $\mathrm{cm}$, 0.05-0.1 mm thick. Hymenial surface cream-colored to pale ochraceous, odontioid; spines densely arranged, 5-6 per mm, up to $0.5 \mathrm{~mm}$ long, rather blunt-pointed, sometimes fusing together and then fimbriate. Margin white to pale cream-colored, adnate, arachnoid, producing abundant thin, short, white hyphal strands. Smooth areas between spines fertile.

Hyphal structure monomitic; hyphae clamped. Subicular hyphae interwoven to subparallel, distinctly thick-walled, branched mostly at sharp angles, (2.6-) 3.2-5.2 (-5.4) $\mu \mathrm{m}$ in diam. $(n=20 / 1)$. Tramal hyphae interwoven to subparallel, distinctly thick-walled, rather loosely arranged, (3.1-) 3.2-4.2 $(-4.8) \mu \mathrm{m}$ in diam. $(n=20 / 1)$, in subhymenium more or less vertically oriented, thin- or slightly thick-walled, mostly shortcelled and slightly inflated, (2.2-) 2.3-3.8 $(-4.1) \mu \mathrm{m}(\mathrm{n}=20 /$ 1). Hyphidia abundant, mostly slightly projecting, simple, 2-3 $\mu \mathrm{m}$ in diam. Basidia suburniform to almost clavate, (1-) 2-4spored, (6.7-) 7.2-12.2 (-12.3) × (3.7-) 3.8-4.8 (-5.0) $\mu \mathrm{m}(\mathrm{n}$ $=20 / 1)$. Basidioles clavate to subglobose, (4.8-) 5.2-7.2 ($7.6) \times(3.5-) 3.8-4.8(-4.9) \mu \mathrm{m}(\mathrm{n}=20 / 1)$. Basidiospores narrowly ellipsoid to broadly cylindrical, straight, a few of the longest spores somewhat fusiform, (3.9-) 4.0-5.3 (-5.4) $\times(2.0-) 2.1-3.0(-3.1) \mu \mathrm{m}(\mathrm{n}=30 / 1), \mathrm{L}=4.51, \mathrm{~W}=2.48, \mathrm{Q}=$ 1.83 .

Remarks. Sistotremastrum denticulatum is morphologically most similar to $S$. aculeocrepitans but it has more densely arranged spines fusing together in characteristic fimbriate groups. Microscopically, these species are almost identical except for loosely arranged and not agglutinated hyphae in $S$. denticulatum. Sistotremastrum fibrillosum has spines not only on the hymenial surface but also on hyphal cords while $S$. denticulatum has sterile hyphal strands. The species is so far known only from the type locality in the southern part of Brazil. 
Sistotremastrum fibrillosum Gruhn \& Alvarado, Phytotaxa 379: 31, 2018-Fig. 10

Basidiocarps effused, soft, covering several $\mathrm{cm}, 0.05-$ $0.1 \mathrm{~mm}$ thick. Hymenial surface cream-colored, odontioid; spines densely arranged, 6-9 per $\mathrm{mm}$, up to $0.4 \mathrm{~mm}$ long, sharp-pointed, often fusing together in fascicles of 2-4, covering also hyphal strands. Margin white to pale cream-colored, floccose or radially fibrillose. Hyphal strands well developed, sometimes a few $\mathrm{cm}$ long and up to $0.5 \mathrm{~mm}$ thick.

Hyphal structure monomitic; hyphae clamped. Subicular hyphae mostly subparallel, distinctly thick-walled, branched mostly at sharp angles, (3.3-) 3.6-6.2 (-7.1) $\mu \mathrm{m}$ in diam. $(\mathrm{n}=$ 20/1); some hyphae encrusted by densely distributed subglobose resinous globules. Tramal hyphae with unevenly thickened walls, rather loosely arranged, subparallel to interwoven, (3.2-) 3.3-4.3 (-4.6) $\mu \mathrm{m}$ in diam. $(\mathrm{n}=20 / 1)$, in subhymenium more or less vertically oriented, thin- or slightly thick-walled, sometimes short-celled and slightly inflated, (1.7-) 2.0-3.2 (-3.6) $\mu \mathrm{m}(\mathrm{n}=20 / 1)$. Hyphidia present, embedded or projecting up to $20 \mu \mathrm{m}$, simple, $1.5-2 \mu \mathrm{m}$ in diam. Basidia suburniform, 4-spored, (9.4-) 10.3-19.2 (-20.8) $\times$ (4.0-) 4.1-4.8 $(-5.1) \mu \mathrm{m}(\mathrm{n}=20 / 1)$. Basidioles clavate to bladder-shaped, 7-10 × 3.5-4.5 $\mu \mathrm{m}$. Basidiospores cylindrical to narrowly ellipsoid, straight, (4.0-) 4.2-5.2 (-5.6) $\times$ (2.1-) 2.2-2.9 (-3.0) $\mu \mathrm{m}(\mathrm{n}=20 / 1), \mathrm{L}=4.72, \mathrm{~W}=2.53, \mathrm{Q}$ $=1.88$, often glued together in large groups.

Remarks. The description above is based on a single specimen from Brazil. Sistotremastrum fibrillosum was originally introduced from four specimens collected in French Guiana (Gruhn et al. 2018). However, available DNA sequences show considerable variation, and this may indicate $S$. fibrillosum in the current sense is a collective species. More material is needed to find a more solid solution. Morphological differences of S. fibrillosum from $S$. aculeocrepitans detected in the same geographic region are treated under the latter species.

Specimens examined. Brazil. Pará: Melgaço, Caxiuanã, ICMBIO headquarter, angiosperm, 13.III.2015 K.H. Larsson 16988* (MG 213771, O).

Fig. 11 Basidiospores of Sistotremastrum spp. a S. geminum (holotype). b S. induratum (holotype). c S. mendax (three left-holotype, three right-Spirin 10060). d $S$. rigidum (holotype). e S. suecicum (holotype). f S. vigilans (three left-Spirin 10097, two right-holotype)
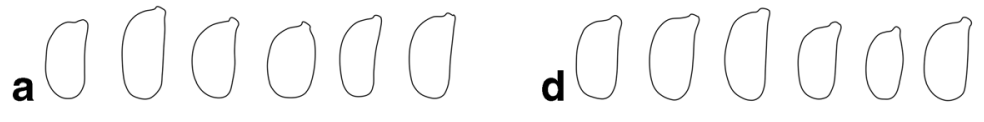

Fig. 12 Microscopic structures of S. geminum (holotype). a Crystals from subhymenial hyphae. b Subhymenial hyphae and hymenial cells. c Subicular hyphae

Sistotremastrum geminum Miettinen \& Spirin, sp. nov.Figs. 11 and 12

MB 833951

Holotype. Indonesia. Papua Barat: Manokwari Reg., Saukorem, Tamuk, $-0.5832^{\circ} 133.14845^{\circ}$, hilly lowland primary rainforest, on an uprooted Intsia bijuga $(150 \mathrm{~cm}$ in diameter, decay stage 1-4/5), 30.X.2010 Miettinen 14333.2* (MAN, isotype-H7009307).

Etymology. Geminus (Lat., adj.)—similar.

Basidiocarps effused, soft-floccose, covering several $\mathrm{cm}$, $0.07-0.1 \mathrm{~mm}$ thick. Hymenial surface pale cream-colored, grandinioid, with irregularly arranged, obtuse warts $7-8$ per $\mathrm{mm}$. Margin pale cream-colored, floccose, partly detaching. Hyphal strands abundant in the underlying substrate, white and richly branched. Smooth areas between warts fertile. 
Hyphal structure monomitic; hyphae clamped. Subicular hyphae interwoven to subparallel, slightly thick-walled, (2.3-) 2.8-4.8 $(-5.2) \mu \mathrm{m}$ in diam. $(\mathrm{n}=20 / 1)$. Tramal hyphae thin-walled, rather loosely arranged, interwoven, as a rule short-celled and more or less distinctly inflated, (3.0-) 3.1$4.8(-6.2) \mu \mathrm{m}$ in diam. $(\mathrm{n}=20 / 1)$. Subhymenial hyphae thinwalled, easily collapsing, often short-celled and slightly inflated or twisted, (2.2-) 2.3-3.4 (-3.7) $\mu \mathrm{m}$ in diam. $(\mathrm{n}=20 / 1)$. Hyphal ends at spine tips thin-walled, short-celled, with occasional short side branches, 3-5 $\mu \mathrm{m}$ in diam., usually encrusted by solitary rhomboid crystals or solid crystalline shields. Cystidia unevenly distributed, thin-walled, varying from obtuse-clavate to subglobose, mostly enclosed, 11-22 × 6.5$9.5 \mu \mathrm{m}$. Hyphidia present, embedded or slightly projecting, simple, $2-3 \mu \mathrm{m}$ in diam. at the apex, sometimes swollen at the base up to $4 \mu \mathrm{m}$ in diam. Basidia suburniform, 4-spored, (7.8-) 8.0-11.2 (-11.8) × (3.6-) 3.7-4.4 (-4.6) $\mu \mathrm{m}(\mathrm{n}=20 /$ 1), senescent basidia slightly thick-walled at the basal part. Basidioles bladder-shaped to subglobose, (4.8-) 4.9-6.8 (7.2) $\times(3.6-) 3.9-4.7(-5.3) \mu \mathrm{m}(\mathrm{n}=20 / 1)$. Basidiospores cylindrical to narrowly ellipsoid, straight to slightly curved, (3.8-) 3.9-5.2 (-5.5) × (1.9-) 2.0-2.6 (-2.9) $\mu \mathrm{m}(\mathrm{n}=30 / 1), \mathrm{L}$ $=4.28, \mathrm{~W}=2.24, \mathrm{Q}=1.92$.

Remarks. Hymenophore with short warts (as opposed to long spines) and thin-walled, loosely arranged tramal hyphae differentiate $S$. geminum from other four-sterigmatic representatives of the genus treated here. The species is so far known only from the type locality in New Guinea.

\section{Sistotremastrum induratum Spirin, sp. nov.-Fig. 11} MB 833952

Holotype. USA. Washington: Pend Oreille Co., Muskegon Lake, $48.7923^{\circ}-117.044^{\circ}$, Abies grandis, 16.X.2014 Spirin 8598* (H 7009717).

Etymology. Induratus (Lat., adj.) — hardening.

Basidiocarps effused, initially pruinose, later compact, covering several $\mathrm{cm}, 0.03-0.1 \mathrm{~mm}$ thick. Hymenial surface whitish to cream-colored, smooth or porulose. Margin first pruinose, later compact and rather sharply delimited. Hyphal strands present in subiculum of vigorously growing specimens, tough and well-differentiated.

Hyphal structure monomitic; hyphae clamped. Hyphae in strands densely arranged and partly agglutinated, more or less parallel, unevenly thick-walled, 5-6 $\mu \mathrm{m}$ in diam. Subicular hyphae interwoven to subparallel, unevenly thick-walled, branched mostly at right angles, (2.7-) $2.8-4.5(-4.7) \mu \mathrm{m}$ in diam. $(n=20 / 1)$. Subhymenial hyphae thin- or slightly thickwalled, densely arranged, some short-celled and slightly inflated, (2.8-) 3.0-4.8 (-5.0) $\mu \mathrm{m}$ in diam. $(\mathrm{n}=40 / 2)$. Hyphidia occasionally present, embedded, simple, $3-5 \mu \mathrm{m}$ in diam. Basidia clavate, 4-6-spored, gradually tapering to the basal part, (13.6-) 14.0-22.8 (-24.0) × (5.1-) 5.2-6.3 (-6.8) $\mu \mathrm{m}$ $(\mathrm{n}=20 / 1)$. Basidioles clavate to bladder-shaped, $(9.8-)$
$10.2-15.9(-16.0) \times(3.8-) 3.9-5.3(-5.7) \mu \mathrm{m}(\mathrm{n}=20 / 1)$. Basidiospores broadly cylindrical to narrowly ovoid, rarely ellipsoid, (4.0-) 4.1-5.3 (-5.8) × (2.5-) 2.6-3.1 (-3.2) $\mu \mathrm{m}$ $(\mathrm{n}=60 / 2), \mathrm{L}=4.37-4.73, \mathrm{~W}=2.79-2.86, \mathrm{Q}=1.53-1.70$.

Remarks. Sistotremastrum induratum has so far been detected in two localities in the North American North-West. It is most similar to the European S. mendax but it has on average shorter basidia and basidiospores, as well as stable differences in ITS sequences. Distribution areas of the two species seem not to overlap.

Specimens examined. USA. Washington: Jefferson Co., Hoh River, Picea sitchensis, 20.X.2014 Spirin 8804*(H).

Sistotremastrum mendax Spirin \& Volobuev, sp. nov.Figs. 5 and 11

MB 833953

Holotype. Norway. Telemark: Nome, Mørkvasslia Nat. Res., 59.288644 $8.865084^{\circ}$, rotten $\log$ of Picea abies, 23.IX.2003 K.H. Larsson $12022 *$ (GB).

Etymology. Mendax (Lat., adj.) - deceptive.

Basidiocarps effused, initially pruinose, later compact, covering several $\mathrm{cm}, 0.05-0.2 \mathrm{~mm}$ thick. Hymenial surface whitish grey to cream-colored or pale ochraceous, smooth or porulose, accidentally cracking only in very old basidiocarps. Margin first pruinose, later compact and rather sharply delimited. Hyphal strands present in subiculum of vigorously growing specimens, normally detectable by the naked eye.

Hyphal structure monomitic; hyphae clamped. Hyphae in strands densely arranged, parallel, slightly thick-walled, 1.5$2.5 \mu \mathrm{m}$ in diam. Subicular hyphae interwoven to subparallel, slightly thick-walled, irregularly branched, (3.1-) 3.7-6.2 (6.3) $\mu \mathrm{m}$ in diam. $(\mathrm{n}=20 / 1)$. Subhymenial hyphae thin- or slightly thick-walled, densely arranged, some short-celled and slightly inflated, (2.2-) 2.3-4.2 (-4.3) $\mu \mathrm{m}$ in diam. $(\mathrm{n}=$ $50 / 3)$. Hyphidia rarely present, embedded, simple, $3-4 \mu \mathrm{m}$ in diam. Basidia clavate, 4-6-spored, gradually tapering to the basal part, (11.4-) 11.7-27.2 (-27.3) × (4.6-) 4.8-6.6 (-7.2) $\mu \mathrm{m}(\mathrm{n}=50 / 3)$. Basidioles clavate to bladder-shaped, $(7.2-)$ 8.2-13.8 (-15.3) × (4.2-) 4.3-6.2 (-6.5) $\mu \mathrm{m}(\mathrm{n}=20 / 2)$. Basidiospores narrowly ellipsoid to broadly cylindrical, some narrowly ovoid, (4.0-) 4.2-7.0 (-7.2) × (2.4-) 2.5-3.5 (-3.6) $\mu \mathrm{m}(\mathrm{n}=180 / 6), \mathrm{L}=475-5.71, \mathrm{~W}=2.76-3.09, \mathrm{Q}=1.71-$ 1.95 .

Remarks. The species is introduced here as a sibling of S. suecicum occurring on wood of Picea in Europe. It differs from S. suecicum s. str. in having broader basidiospores and much better developed and easily observable hyphal strands in subiculum. Moreover, mature basidiocarps of $S$. suecicum are cracking in a characteristic way while they mostly stay continuous in $S$. mendax (Fig. 2). The host preference alone is not enough to recognize these species because $S$. suecicum sometimes occurs on spruce, as well as other coniferous trees. The 
North American $S$. induratum has on average shorter basidia and basidiospores than $S$. mendax (Table 2).

According to our observations, S. mendax prefers oldgrowth spruce-dominated forests subjected to minimal (if any) logging activity. Further ecological studies are needed to confirm these observations.

Specimens examined. Czech Republic. South Bohemia Reg.: Boubín Nat. Res., P. abies, 29.X.2011 Vlasák 1110/14.1 (H). Finland. Etelä-Karjala: Miehikkälä, Kaitai, P. abies, 3.VIII.1976 Fagerström (H). Satakunta: Lamppi, P. abies, 31.VIII.1939 Laurila (H). Norway. Sør-Trøndelag: Neadalen, Tydal, P. abies, 24.IX.2011 J. Nordén 9579* (O). Poland. Podlasie: Hajnówka, Bialowieza NP, P. abies, 23.IX.2019 Miettinen 22476*(H). Russia. Karelia: Medvezhiegorsk Dist., Krivozero, P. abies, 7.VI.1942 Laurila 3251a, 3258 (H). Leningrad Reg.: Boksitogorsk Dist., Chagoda, P. abies, 9.V.2018 Spirin 11887 (H), Kolp', P. abies, 8.V.2016 Spirin 10057, 10060 (H), 9.V.2016 Spirin 10086 (H); Podporozhie Dist., Oksboloto, P. abies, 27.V.2017 Spirin 11223 (H), Vazhinka, P. abies, 21.V.2016 Spirin 10107 (H), 4.VI.2016 Spirin 10151 (H), 21.VI.2016 Spirin 10226 (H), 26.V.2017 Spirin 11208 (H), Miettinen 20946* (H), 14-15.X.2017 Spirin 11699, $11738(\mathrm{H})$. Sweden. Värmland: Torsby, P. abies, 5.X.2010 J. Nordén $7350(\mathrm{O})$.

Sistotremastrum rigidum Motato-Vásquez \& Spirin, sp. nov.-Fig. 11

MB 833954

Holotype. Brazil. Saõ Paulo: Trilha do Mirante, Serra do Mar State Park, $-23.983^{\circ}-46.7467^{\circ}$, fallen log, 17.XI.2016 Motato-Vásquez 833* (SP 466010, isotype-H).

Etymology. Rigidus (Lat., adj.) — rigid, tough.

Basidiocarps effused, crustaceous, tough, covering several $\mathrm{cm}, 0.05-0.08 \mathrm{~mm}$ thick. Hymenial surface almost white to cream-colored, smooth. Margin white, indistinctly fimbriate, adnate, occasionally with short hyphal strands.

Hyphal structure monomitic; hyphae clamped. Subicular hyphae tightly glued together, interwoven to subparallel, thick-walled, hardly visible, $2-4 \mu \mathrm{m}$ in diam. Subhymenial hyphae slightly to distinctly thick-walled, densely arranged and partly glued together, some short-celled and slightly inflated, (2.0-) 2.1-3.0 (-3.2) $\mu \mathrm{m}$ in diam. $(\mathrm{n}=20 / 1)$. Hyphidia rarely present, mostly embedded, simple, $2-2.5 \mu \mathrm{m}$ in diam. Basidia suburniform to almost clavate, 4-spored, (7.7-) 9.2$14.3(-16.2) \times(4.6-) 4.8-6.6(-7.2) \mu \mathrm{m}(\mathrm{n}=20 / 1)$, senescent basidia slightly thick-walled at the basal part. Basidioles rare, subglobose, 5-6 $\times 4-5 \mu \mathrm{m}$. Basidiospores cylindrical to broadly cylindrical, $(3.8-) 4.0-5.1(-5.2) \times 2.0-2.5(-2.7)$ $\mu \mathrm{m}(\mathrm{n}=30 / 1), \mathrm{L}=4.44, \mathrm{~W}=2.22, \mathrm{Q}=2.01$.

Remarks. Basidiocarps of $S$. rigidum are exceptionally tough due to densely arranged and partly agglutinated hyphae, making the structure resemble species of Phlebia s.l. However, none of the latter ones forms hyphal strands or has suburniform basidia with slightly but distinctly thickened walls. Another four-sterigmatic species with smooth hymenophore, S. confusum, occurs in Brazil. However, it differs from S. rigidum in having soft basidiocarps and larger basidia and basidiospores.

Sistotremastrum suecicum Litsch. ex J. Erikss., Symb. Bot. Upsalienses 16 (1): 62, 1958-Figs. 5 and 11

Holotype. Sweden. Upland: Uppsala, Bondkyrka, "on the under-side of coniferous fencing-material, lying in a wet spot" [Pinus sylvestris - identified by Pirkko Harju, H], 4.I.1933 Lundell (Fungi Exsiccati Suecici \#464) (S F204406, studied).

Basidiocarps effused, initially pruinose, later waxy, covering several $\mathrm{cm}, 0.05-0.2 \mathrm{~mm}$ thick. Hymenial surface whitish to cream-colored or pale ochraceous, smooth or porulose, in older basidiocarps often cracking into small irregular patches. Margin first pruinose, later compact and rather sharply delimited. Hyphal strands present in basal layer of vigorously growing specimens.

Hyphal structure monomitic; hyphae clamped. Hyphae in strands densely arranged, parallel, slightly thick-walled, 1.5$2.5 \mu \mathrm{m}$ in diam. Subicular hyphae interwoven to subparallel, slightly thick-walled, branched mostly at right angles, (3.8-) 3.9-5.8 (-6.2) $\mu \mathrm{m}$ in diam. $(\mathrm{n}=40 / 2)$. Subhymenial hyphae thin- or slightly thick-walled, densely and randomly arranged, in subhymenium more or less vertically oriented, some shortcelled and slightly inflated, (2.2-) 2.3-4.1 (-4.2) $\mu \mathrm{m}$ in diam. ( $n=100 / 5)$. Hyphidia occasionally present, embedded or projecting (especially in young specimens) up to $15 \mu \mathrm{m}$ above hymenial layer, simple, 2-4 $\mu \mathrm{m}$ in diam. Basidia clavate, 4-6spored, gradually tapering to the basal part, (10.6-) 12.8-26.7 $(-26.8) \times(4.1-) 4.2-6.2(-6.3) \mu \mathrm{m}(\mathrm{n}=80 / 5)$. Basidioles clavate to bladder-shaped, (8.3-) 8.6-14.2 (-15.1) $\times(3.3-)$ 3.8-5.2 $(-5.8) \mu \mathrm{m}(\mathrm{n}=20 / 1)$. Basidiospores cylindrical to narrowly ovoid, (4.1-) 4.2-6.2 (-6.3) × (2.1-) 2.2-3.1 (3.2) $\mu \mathrm{m}(\mathrm{n}=180 / 6), \mathrm{L}=4.56-5.55, \mathrm{~W}=2.42-2.80, \mathrm{Q}=$ 1.83-2.06.

Remarks. Sistotremastrum suecicum was described from Sweden (Eriksson 1958) and has so far been treated in a collective sense, i.e., the name has been used for all collections from coniferous hosts. According to our results, S. suecicum occurs almost exclusively on Pinus spp., like the so-called kelo trees in North Europe (Niemelä et al. 2002). It is considered an indicator species of old pine forests of high conservation value in Finland (Niemelä 2016). Most specimens from other gymnosperm hosts belong either to $S$. mendax (in Europe) or to $S$. induratum (in the North American North-West). Differences of $S$. suecicum from the latter two species are discussed under $S$. mendax. The species forms basidiocarps throughout the year. 
An ITS sequence of $S$. suecicum UC2022903 found on pine wood in California (GenBank KP814148) is clearly different from the rest of the pine-dwelling collections and seemingly representing a species of its own. We leave this problem until more material from the US Pacific will be available. Future studies should elucidate if $S$. suecicum s. str. is also present in this geographic area or if its distribution in North America is limited to the east of the Rocky Mountains.

Specimens examined. Canada. British Columbia: FraserFort George Reg. Dist., Jackman Flats Prov. Park, Pinus contorta, 25.VII.2015 Spirin 8932* (H). Nova Scotia: Kentville, Ravine, Pinus resinosa, 8.VIII.1954 Harrison (H ex DAOM 52237). Finland. Varsinaissuomi: Parainen, Kuggö, P. sylvestris, 24.X.2009 Kunttu 5959* (H). PohjoisSavo: Heinävesi, Vihtarinniemi, P. sylvestris, 2.X.2006 Miettinen 11044.1* (H). Inarin Lappi: Inari, Piekanavaara, P. sylvestris, 28.VIII.2008 Miettinen 13310.1 (H). Russia. Arkhangelsk Reg.: Krasnoborsk Dist., P. sylvestris, 5.VIII.2013 Kotkova (LE 295792*). Krasnoyarsk Reg.: Turukhansk Dist., Bor, P. sylvestris, 24.VIII.2013 Kotiranta 26451 (H). Leningrad Reg.: Boksitogorsk Dist., Chagoda, P. sylvestris, 10.V.2018 Spirin 11888, $11890(\mathrm{H})$, Shidrozero, P. sylvestris, 14.VIII.2014 Spirin 6974 (H); Podporozhie Dist., Kurba, P. sylvestris, 19.IX.2009 Spirin 3236 (H), Vazhinka, P. abies, 14.X.2017 Spirin 11697 (H). Nizhny Novgorod Reg.: Arzamas Dist., Pustyn', P. sylvestris, 13.VIII.2015 Spirin 9227, 9233 (H). Sweden. Jämtland: Härjedalen, Glumtjärnarna, P. sylvestris, 14.VIII.2011 Miettinen 14550.1* (H). Ångermanland: Resele, Vignäsbodarna, P. abies (?), 28.VIII.2002 K.H. Larsson 11849* (GB). Jämtland: Härjedalen, Ransjön, P. sylvestris, 14.VIII.2011 Miettinen 14548 (H). USA. Massachusetts: Worcester Co., Holden, fallen branch, 26.IX.2011 Miettinen 14829*(H), Tsuga canadensis, 21.IV.2013 Miettinen 16061* (H), Tsuga/Pinus sp., 27.VII.2013 Miettinen 16618*(H).

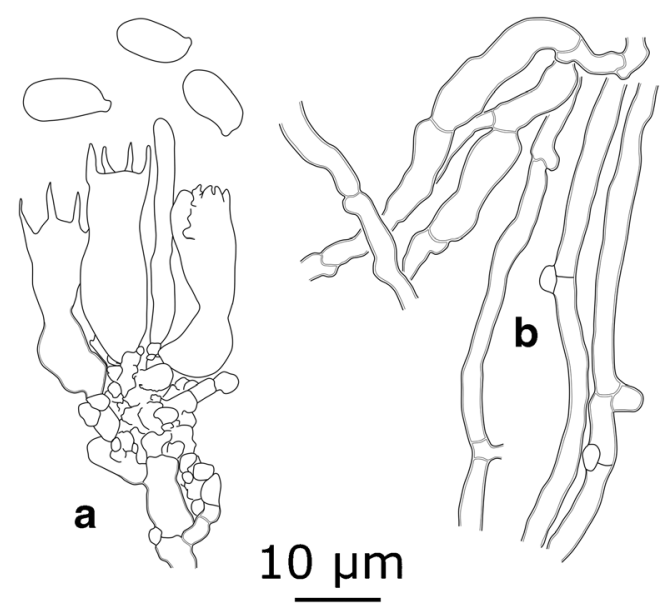

Fig. 13 Microscopic structures of S. vigilans (holotype). a Subhymenial hyphae and hymenial cells. b Subicular hyphae
Sistoremastrum vigilans K.H. Larss. \& Spirin, sp. nov.Figs. 11 and 13

MB 833955

Holotype. Norway. Vestfold: Stokke, Melsomvik, 59.22541 ${ }^{\circ} 10.35202^{\circ}$, rotten log of Picea abies, 30.IV.2011 Fonneland 2011-78*(O).

Etymology. Vigilans (Lat., adj.)—vigilant.

Basidiocarps effused, compact, covering several cm, 0.05$0.2 \mathrm{~mm}$ thick. Hymenial surface white to cream-colored or pale ochraceous, first smooth, in older basidiocarps irregularly cracking and unevenly tuberculate. Margin first pruinose, later compact and sharply delimited. Hyphal strands often present, well-developed, white, occurring in subiculum and sometimes in the underlying substrate.

Hyphal structure monomitic; hyphae clamped. Subicular hyphae in parallel bundles or interwoven, with unevenly thickened walls, randomly branched, some tortuous, with occasional oil-drops, (3.1-) 3.2-5.6 (-5.7) $\mu \mathrm{m}$ in diam. $(n=60 /$ 3 ), in older parts with bubble-like inflations 7-10 $\mu \mathrm{m}$ in diam. Subhymenial hyphae thin- or slightly thick-walled, densely arranged, some short-celled and slightly inflated, (2.0-) 2.2$4.2(-5.0) \mu \mathrm{m}$ in diam. $(n=60 / 3)$. Hyphidia occasionally present, embedded or projecting up to $20 \mu \mathrm{m}$, simple or with accidental side branches, $1.5-5 \mu \mathrm{m}$ in diam. Basidia clavate to utriform, 4-spored, with widened or tapering bases, (11.3-) 11.4-34.1 (-38.2) × (5.3-) 5.4-8.0 (-8.1) $\mu \mathrm{m}(\mathrm{n}=50 / 3)$. Basidiospores narrowly ellipsoid to long cylindrical, straight or slightly curved, a few lacrymoid or sigmoid, (6.1-) 6.3$11.2(-11.4) \times(3.0-) 3.1-5.4(-6.2) \mu \mathrm{m}(\mathrm{n}=150 / 5), \mathrm{L}=7.43-$ 9.95, $\mathrm{W}=3.64-4.69, \mathrm{Q}=1.81-2.22$.

Remarks. Sistotremastrum vigilans is the only foursterigmatic species of the genus so far detected in temperate and boreal forests of the Northern Hemisphere. It occurs on coniferous hosts, either late in the season or in spring, and this might have been a reason for overlooking it. The species is rare and tends to occur in rather old forests with large-diameter dead wood.

Sistotremastrum vigilans has the largest basidiospores in the genus and thus it can be mistaken for a Hyphoderma s. 1. species. Basidia of highly variable size, basidiospores with homogeneous contents (not guttulate as in Hyphoderma) and slightly thicker walls, as well as occasionally inflated subicular hyphae are the most reliable traits for a morphological identification of this species. The well-developed hyphal strands (if present) are another clue to recognizing S. vigilans.

Specimens examined. Canada. Québec: Outaouais, Gatineau Nat. Park, Abies balsamea, 14.X.1967 Eriksson 9106 (GB, K), conifer, 15.X.1967 Eriksson 9140, 9270, 9279 (GB, K), A. balsamea, 22.X.1967 Eriksson 9281 (GB, K). Finland. Uusimaa: Helsinki, Laajasalo, P. abies, 25.XI.1934 Häyrén (H), Haltiala, P. abies, 20.XII.2020 Miettinen $24513(\mathrm{H})$. Satakunta: Vuorijärvi, P. abies, 6.IX.1937 Laurila (H). Norway. Vestfold: Stokke, 
Melsomvik, P. abies, 30.IV.2011 Fonneland 2011-77 (O). Russia. Leningrad Reg.: Podporozhie Dist., Vazhinka, P. abies, 21.V.2016 Spirin 10097* (H), 22.V.2016 Spirin 10135 (H). USA. Washington: Jefferson Co., Hoh River, Tsuga heterophylla, 20.X.2014 Spirin 8778*(H).

\section{Discussion}

In this paper, we present the most comprehensive study of Sistotremastrum to date. Sistotremastrum sensu lato now holds 18 species, which means that species number more than doubled. The original concept of Sistotremastrum (Eriksson 1958) contained only two species, S. suecicum and $S$. niveocremeum. However, with the much richer sampling available to us, the phylogenetic analyses prompted us to segregate the species around $S$. niveocremeum into the new genus Sertulicium.

Larsson (2007) showed that Sistotremastrum suecicum and $S$. niveocremeum belong in the Trechisporales forming a strongly supported sister clade to the rest of the order. Larsson (2007) named this clade the Sistotremastrum family while for the sister clade the name Hydnodontaceae is available. The same strongly supported topology was recovered by Gruhn et al. (2018) in a paper where two new Sistotremastrum species were introduced. In the present work, we were not able to generate high support values for the node connecting Sistotremastrum and Sertulicium (Fig. 1). The reason for this is unclear but probably depends on the generally high diversity of the nuclear ribosomal markers in Trechisporales. We think it is likely that the concept of Sistotremastrum family as defined by Larsson will survive, but the question can only be solved with sequences from an expanded set of genes.

Three species, Sistotremastrum denticulatum, S. geminum, and $S$. rigidum, are described based on single specimens. While we normally would avoid doing this, we found it justified in these cases. These three species are all well separated in ITS alignments and differs by $3.6 \%, 4.6 \%$, and $9.0 \%$, respectively, from their phylogenetically closest relatives (Fig. 1). Furthermore, all the close relatives of these single-specimen species are represented by multiple ITS sequences, increasing confidence that such big ITS differences represent inter- and not infraspecific variation. All these species are also morphologically clearly separable from their closest relatives. Lastly, we used distinct distribution areas as a criterion. Distribution areas of $S$. denticulatum and S. rigidum do not overlap with their closest relatives. Difference in distribution areas is not as clear in the case of $S$. geminum and its closest relative $S$. aculeatum, which are both found in South-East Asia. However, the latter has been found so far only west of Wallace's line (Sumatra and Asian mainland), while $S$. geminum east of it, in Australasia (New Guinea).

\section{Other specimens examined}

Brevicellicium exile. USA. Washington: Clark Co., Gifford Pinchot Nat. Forest, T. heterophylla, 11.X.2014 Spirin 8370* $(\mathrm{H})$.

B. olivascens. Russia. Nizhny Novgorod Reg.: Bogorodsk Dist., Krastelikha, Q. robur, 5.IX.2011 Spirin 4446* (H). Sweden. Bohuslän: Restenäs, Ulvesund, decaying angiosperm branch, 3.X.1998, Larsson 8571* (GB).

B. viridulum. Russia. Sakhalin: Uglegorsk Dist., Telnovskoe, Alnus hirsuta, 1.IX.2007 Kotiranta 29271* (H).

Luellia cystidiata. Denmark. Jylland: Varde, Bordrup Klitplantage, P. abies, 6.X.2009 Petersen \& Lassøe 13875 (GB).

Paullicorticium allantosporum. Norway. Hedmark: Løten, Gitvola, P. abies, 26.IX.2018 Spirin 12413 (H).

Pteridomyces galzinii. Italy. Tuscany: Arezzo, Camaldoli Nat. Res., Polystichum aculeatum, 17.XI.2010 Bernicchia $8122 *(\mathrm{~GB})$.

Sertulicium aff. granuliferum. Kenya. Eastern Prov.: Meru, Chigora, decayed wood, 31.I.1973 Ryvarden 3593 (O); Nyeri, Regati, decayed wood, 2.II.1973 Ryvarden 9777 (O), Ryvarden 9888 (O). Tanzania. Tanga Prov.: Tanga Dist., Amani, decayed wood, 18.II.1973 Ryvarden 10575 (O, H).

Sertulicium sp. 1. Russia. Khabarovsk Reg.: Khabarovsk Dist., Ilga, Corylus mandshurica, 11.VIII.2012 Spirin 5158* (H).

Sertulicium sp. 2. Sweden. Bohuslän: Säve, P. sylvestris (bark), 4.III.1973 Arvidsson (GB).

Suillosporium cystidiatum. Russia. Khabarovsk Reg.: Solnechnyi Dist., Igdomi, Picea ajanensis, 5.VIII.2011 Spirin 3830* (H).

Acknowledgements Greet Van Autgaerden (Antwerpen, Belgium), Eugeny Dunaev (Young Naturalist Club of the Zoological Museum, Moscow University, Russia), and Alexander Ordynets (Kassel, Germany) kindly provided us with valuable fungal collections. Serena Lee organized and participated in field work in Singapore. Pirkko Harju (Helsinki, Finland) identified host trees of some important specimens.

Author contribution All authors contributed to the study conception and design. Material preparation, data collection, and analysis were initially performed by Viacheslav Spirin, Sergey Volobuev, Ilya Viner, Otto Miettinen, and Karl-Henrik Larsson. The authors Josef Vlasák, Nathan Schoutteten, Viviana Motato-Vásquez, Heikki Kotiranta, and Hernawati provided further important material and/or sequences. The first draft of the manuscript was written by Viacheslav Spirin and all authors commented on previous versions of the manuscript. All authors read and approved the final manuscript.

Funding Open access funding provided by University of Helsinki including Helsinki University Central Hospital. Komarov Botanical Institute RAS (institutional research project "Biodiversity, ecology, structural and functional features of fungi and fungus-like protists", AAAA-A19119020890079-6, with equipment of The Core Facilities Center "Cell and Molecular Technologies in Plant Science") (St. Petersburg, Russia) (the 
author SV); the grant of the President of the Russian Federation (project MK-3216.2019.11) (the author SV); Societas pro Fauna et Flora Fennica (the author IV); Academy of Sciences of the Czech Republic (project number RVO: 60077344) (the author JV); Flemish Research Council (FWO) (grant number 11E0420N) (the author NS); Coordenação de Aperfeiçoamento Pessoal de Nível Superior-CAPES (Brazil) (the author VMV); The Rufford Small Grants Foundation (the author VMV).

Data availability DNA sequences used in the present study are available in GenBank. Alignments were deposited in TreeBASE. Fungal specimens are stored in public herbaria (as indicated under "Specimens examined").

\section{Declarations}

\section{Ethics approval Not applicable}

Conflict of interest The authors declare no competing interests.

Open Access This article is licensed under a Creative Commons Attribution 4.0 International License, which permits use, sharing, adaptation, distribution and reproduction in any medium or format, as long as you give appropriate credit to the original author(s) and the source, provide a link to the Creative Commons licence, and indicate if changes were made. The images or other third party material in this article are included in the article's Creative Commons licence, unless indicated otherwise in a credit line to the material. If material is not included in the article's Creative Commons licence and your intended use is not permitted by statutory regulation or exceeds the permitted use, you will need to obtain permission directly from the copyright holder. To view a copy of this licence, visit http://creativecommons.org/licenses/by/4.0/.

\section{References}

Benson DA, Cavanaugh M, Clark K, Karsch-Mizrachi I, Ostell J, Pruitt KD, Sayers EW (2018) GenBank. Nucleic Acids Res 46(D1):D41D47. https://doi.org/10.1093/nar/gkx1094

Boidin J, Gilles G (1990) Corticiés s.l. interessantes ou nouveax pour la France (Basidiomycotina). Bull Soc Mycol France 106:135-167

Boidin J, Gilles G (1994) Contribution à la connaissance des Corticiés à basides urniformes ou suburniformes (Basidiomycotina). Bull Soc Mycol France 110:185-229

Eriksson J (1958) Studies in the Heterobasidiomycetes and Homobasidiomycetes - Aphyllophorales of Muddus National Park in North Sweden. Symb Bot Upsal 16:1-172

Eriksson J, Ryvarden L (1975) The Corticiaceae of North Europe. 3. Fungiflora, Oslo

Eriksson J, Hjortstam K, Ryvarden L (1978) The Corticiaceae of North Europe. 5. Fungiflora, Oslo

Eriksson J, Hjortstam K, Ryvarden L (1984) The Corticiaceae of North Europe. 7. Fungiflora, Oslo

Gardes M, Bruns TD (1993) ITS primers with enhanced specificity for basidiomycetes application to the identification of mycorrhizae and rusts. Mol Ecol 2:132-118. https://doi.org/10.1111/j.1365-294x. 1993.tb00005.x

Gouy M, Guindon S, Gascuel O (2010) SeaView version 4: a multiplatform graphical user interface for sequence alignment and phylogenetic tree building. Mol Biol Evol 27(2):221-224. https:// doi.org/10.1093/molbev/msp259

Gruhn G, Alvarado P, Hallenberg N, Roy M, Courtecuisse R (2018) Contribution to the taxonomy of Sistotremastrum (Trechisporales,
Basidiomycota) and the description of two new species, S. fibrillosum and S. aculeocrepitans. Phytotaxa 379:27-38

Hallenberg N (1978) Wood-fungi (Corticiaceae, Coniophoraceae, Lachnocladiaceae, Thelephoraceae) in North Iran. I. Iranian J Plant Pat 14:38-87

Haynes KA, Westerneng TJ, Fell JW, Moens W (1995) Rapid detection and identification of pathogenic fungi by polymerase chain reaction amplification of large subunit ribosomal DNA. J Med Vet Mycol 33(5):319-325. https://doi.org/10.1080/02681219580000641

Huffman JA, Prenni AJ, DeMott PJ, Pöhlker C, Mason RH, Robinson NH, Fröhlich-Nowoisky J, Tobo Y, Després VR, Garcia E, Gochis DJ, Harris E, Müller-Germann I, Ruzene I, Schmer B, Sinha B, Day DA, Andreae MO, Jimenez JL, Gallagher M, Kreidenweis SM, Bertram AK, Pöschl U (2013) High concentration of biological aerosol particles and ice nuclei during and after rain. Atmos Chem Phys 13:6151-6164. https://doi.org/10.5194/acp-13-6151-2013

Jülich W (1982) Notes on some Basidiomycetes (Aphyllophorales and Heterobasidiomycetes). Persoonia 11(4):421-428

Katoh K, Rozewicki J, Yamada KD (2017) MAFFT online service: multiple sequence align-ment, interactive sequence choice and visualization. Brief Bioinform: bbx108. https://doi.org/10.1093/bib/ bbx 108

Kearse M, Moir R, Wilson A, Stones-Havas S, Cheung M, Sturrock S, Buxton S, Cooper A, Markowitz S, Duran C, Thierer T, Ashton B, Meintjes P, Drummond A (2012) Geneious Basic: an integrated and extendable desktop software platform for the organization and analysis of sequence data. Bioinformatics 28(12):1647-1649. https:// doi.org/10.1093/bioinformatics/bts199

Larsson KH (1992) The genus Trechispora. University of Göteborg, Göteborg

Larsson KH (2001) The position of Poria mucida inferred from nuclear ribosomal DNA sequences. Harvard Papers Bot 6:131-138

Larsson KH (2007) Re-thinking the classification of corticioid fungi. Mycol Res 111:1040-1063

Larsson KH, Larsson E, Kõljalg U (2004) High phylogenetic diversity among corticioid homobasidiomycetes. Mycol Res 108:983-1002

Liu SL, Ma HX, He SH, Dai YC (2019) Four new corticioid species in Trechisporales (Basidiomycota) from East Asia and notes on phylogeny of the order. MycoKeys 48:97-113

Menkis A, Allmer J, Vasiliauskas R, Lygis V, Stenlid J, Finlay R (2004) Ecology and molecular characterization of dark septate fungi from roots, living stems, coarse and fine woody debris. Mycol Res 108: 965-973

Miettinen O, Vlasák J, Rivoire B, Spirin V (2018) Postia caesia complex (Polyporales, Basidiomycota) in temperate Northern Hemisphere. Fungal Syst Evol 1:101-129

Milne I, Lindner D, Bayer M, Husmeier D, McGuire G, Marshall DF, Wright F (2008) TOPALi v2: a rich graphical interface for evolutionary analyses of multiple alignments on HPC clusters and multicore desktops. Bioinformatics 25(1):126-127. https://doi.org/10. 1093/bioinformatics/btn575

Nagy LG, Riley R, Tritt A, Adam C, Daum C, Floudas D, Sun H, Yadav JS, Pangilinan J, Larsson K-H, Matsuura K, Barry K, Labutti K, Kuo R, Ohm RA, Bhattacharya SS, Shirouzu T, Yoshinaga Y, Martin FM, Grigoriev IV, Hibbett DS (2016) Comparative genomics of early-diverging mushroom-forming fungi provides insights into the origins of lignocellulose decay capabilities. Mol Biol Evol 33(4):959-970. https://doi.org/10.1093/molbev/msv337

Niemelä T (2016) Suomen käävät. Norrlinia 31:1-430

Niemelä T, Wallenius T, Kotiranta H (2002) The kelo tree, a vanishing substrate of specified wood-inhabiting fungi. Polish Botanical Journal 47:91-101

Nilsson RH, Larsson K-H, Taylor AFS, Bengtsson-Palme J, Jeppesen TS, Schigel D, Kennedy P, Picard K, Glöckner FO, Tedersoo L, Saar I, Kõljalg U, Abarenkov K (2018) The UNITE database for molecular identification of fungi: handling dark taxa and parallel taxonomic 
classifications. Nucleic Acids Res 47(D1):D259-D264. https://doi. org/10.1093/nar/gky1022

Oberwinkler F (1965) Primitive Basidiomyceten. Revision einiger Formenkreise von Basidienpilzen mit plastischer Basidie. Sydowia 19:1-72

Ronquist F, Teslenko M, Van Der Mark P, Ayres DL, Darling A, Höhna S, Larget B, Liu L, Suchard M, Huelsenbeck JP (2012) MrBayes 3.2: efficient Bayesian phylogenetic inference and model choice across a large model space. Syst Biol 61:539-542. https://doi.org/ $10.1093 /$ sysbio/sys029

Stamatakis A (2006) Raxml-vi-hpc: maximum likelihood-based phylogenetic analyses with thousands of taxa and mixed models. Bioinformatics 22:2688-2690. https://doi.org/10.1093/ bioinformatics/btl446

Tamura K, Stecher G, Peterson D, Filipski A, Kumar S (2013) MEGA6: molecular evolutionary genetics analysis version 6.0. Mol Biol Evol 30:2725-2729. https://doi.org/10.1093/molbev/mst197

Telleria MT, Melo I, Dueñas M, Salcedo I, Beltrán-Tejera E, RodríguezArmas JL, Martín MP (2013a) Sistotremastrum guttuliferum: a new species from the Macaronesian islands. Mycol Progress 12:687-692
Telleria MT, Melo I, Dueñas M, Larsson KH, Paz Martín MP (2013b) Molecular analyses confirm Brevicellicium in Trechisporales. IMA Fungus 4:21-28

Telleria MT, Dueñas M, Martín MP (2014) A new species of Sistotremastrum (Basidiomycota, Trechisporales) from Huinay Reserve. Phytotaxa 158:93-98

Thiers B (2019) Index Herbariorum: a global directory of public herbaria and associated stuff [continuosly updated]. New York Botanical Garden's Virtual Herbarium. http://sweetgum.nybg.org/ih

Vilgalys R, Hester M (1990) Rapid genetic identification and mapping of enzymatically amplified ribosomal DNA from several species of Cryptococcus. J Bacteriol 172:4238-4246

Wu F, Yuan Y, Zhao CL (2015) Porpomyces submucidus (Hydnodontaceae, Basidiomycota), a new species from tropical China based on morphological and molecular evidence. Phytotaxa 230:61-68

Publisher's note Springer Nature remains neutral with regard to jurisdictional claims in published maps and institutional affiliations. 